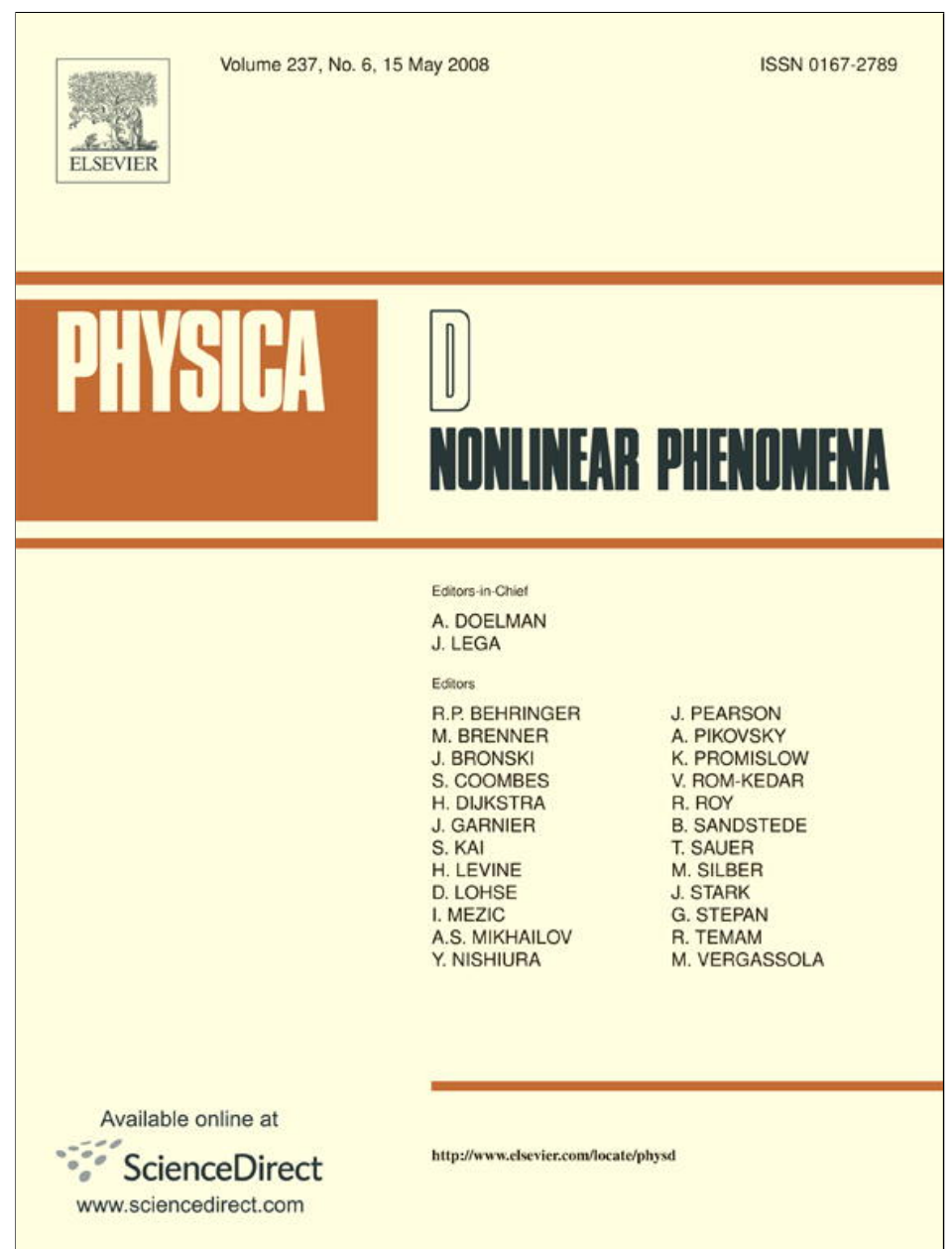

This article appeared in a journal published by Elsevier. The attached copy is furnished to the author for internal non-commercial research and education use, including for instruction at the authors institution and sharing with colleagues.

Other uses, including reproduction and distribution, or selling or licensing copies, or posting to personal, institutional or third party websites are prohibited.

In most cases authors are permitted to post their version of the article (e.g. in Word or Tex form) to their personal website or institutional repository. Authors requiring further information regarding Elsevier's archiving and manuscript policies are encouraged to visit:

http://www.elsevier.com/copyright 


\title{
Two-dimensional Euler flows in slowly deforming domains
}

\author{
J. Vanneste ${ }^{\mathrm{a}}$, D. Wirosoetisno ${ }^{\mathrm{b}, *}$ \\ a School of Mathematics and Maxwell Institute for Mathematical Sciences, University of Edinburgh, Edinburgh EH9 $3 J Z$, UK \\ ${ }^{\mathrm{b}}$ Department of Mathematical Sciences, University of Durham, Durham DH1 3LE, UK \\ Received 28 November 2006; received in revised form 10 August 2007; accepted 26 October 2007 \\ Available online 7 November 2007 \\ Communicated by M. Vergassola
}

\begin{abstract}
We consider the evolution of an incompressible two-dimensional perfect fluid as the boundary of its domain is deformed in a prescribed fashion. The flow is taken to be initially steady, and the boundary deformation is assumed to be slow compared to the fluid motion. The Eulerian flow is found to remain approximately steady throughout the evolution. At leading order, the velocity field depends instantaneously on the shape of the domain boundary, and it is determined by the steadiness and vorticity-preservation conditions. This is made explicit by reformulating the problem in terms of an area-preserving diffeomorphism $g_{\Lambda}$ which transports the vorticity. The first-order correction to the velocity field is linear in the boundary velocity, and we show how it can be computed from the time derivative of $g_{\Lambda}$.

The evolution of the Lagrangian position of fluid particles is also examined. Thanks to vorticity conservation, this position can be specified by an angle-like coordinate along vorticity contours. An evolution equation for this angle is derived, and the net change in angle resulting from a cyclic deformation of the domain boundary is calculated. This includes a geometric contribution which can be expressed as the integral of a certain curvature over the interior of the circuit that is traced by the parameters defining the deforming boundary.

A perturbation approach using Lie series is developed for the computation of both the Eulerian flow and geometric angle for small deformations of the boundary. Explicit results are presented for the evolution of nearly axisymmetric flows in slightly deformed discs.
\end{abstract}

(c) 2007 Elsevier B.V. All rights reserved.

Keywords: Adiabatic invariance; Geometric angle; 2d Euler

\section{Introduction}

This paper examines the dynamics of a two-dimensional (2d) fluid inside a container whose boundary is deformed slowly. The fluid is assumed to be perfect and incompressible; consistent with the latter assumption, the area of the container is constant. Beyond potential applications such as the control of fluid flows, we use the problem as a paradigm for the study of Hamiltonian fluid models depending on slowly-varying parameters. This is an obvious first step: the 2d Euler equations governing incompressible perfect fluids are indeed Hamiltonian (e.g., [17,19]), and imposing boundary deformations is arguably the most natural way of introducing a parameter dependence. As is well known in finite dimensions, Hamiltonian systems are strongly constrained; as a result, slow changes of parameters lead to a remarkable behaviour encapsulated in the theory of adiabatic invariance (see, e.g., [3,12]) and geometric angles [9,5]. In 2d Euler, the material invariance of vorticity (e.g., [18]) similarly imposes a strong constraint on the system, which we exploit extensively to derive what can be interpreted as fluid-dynamical versions of adiabatic invariance and geometric angle.

\footnotetext{
* Corresponding address: Department of Mathematical Sciences, Science Laboratories, University of Durham, South Road, Durham DH1 3 LE, UK. Tel.: +44 191334 3115; fax: +44 1913343051.

E-mail addresses: j.vanneste@ed.ac.uk (J. Vanneste), djoko.wirosoetisno@durham.ac.uk (D. Wirosoetisno).
} 
The problem we consider here is rather involved in its full generality. To make progress, we make a number of assumptions and consider the following scenario. At an initial time, a steady flow is given in some simply-connected domain $D_{0}$. The streamlines have the simplest topology, that of nested closed curves, and the flow is Arnold stable (see Section 2 below). We then assume that this continues to hold throughout the evolution as the domain is being deformed. With these hypotheses, we use an asymptotic approach, based on the separation between the timescale of the boundary deformation and that of the flow, characterised by a small parameter $\varepsilon$. We consider the dynamics over timescales of $O(1 / \varepsilon)$ so that deformations of order one are achieved. We ask two questions: (i) what is the (Eulerian) flow at any time; and (ii) what is the (Lagrangian) position of the fluid particles?

The first question is answered by first showing that the leading-order flow is steady at all times. We then recast the problem in terms of an area-preserving diffeomorphism $g_{\Lambda}$ mapping the vorticity in the initial domain $D_{0}$ to the leading-order vorticity in the deformed domain. This diffeomorphism coincides with that studied in a previous paper [25, henceforth WV]. In that paper, we considered a static problem, regarding steady solutions of the $2 \mathrm{~d}$ Euler equations as fixed points of a dynamical system, and examining their persistence as the parameters defining the shape of their domain are varied. The persistence of steady flows was established under certain hypotheses by showing that a diffeomorphism analogous to $g_{\Lambda}$ exists and is unique. This existence and uniqueness can be used here to show that, at any stage in the deformation, the leading-order flow is completely determined by the instantaneous shape of the boundary (i.e. it is independent of the history of past shapes). We gain further insight into this property by examining the effect of infinitesimal domain deformations. This is naturally described using the language of differential forms: in particular, a (connection) one-form, expressing changes in $g_{\Lambda}$ associated with infinitesimal domain deformations, is given a central role in this paper.

We also go beyond the leading-order approximation to the flow and examine formally higher-order approximations. Of particular interest is the first-order, $O(\varepsilon)$ correction, which is linear in the boundary velocity and admits an interesting geometric interpretation. The higher-order approximations are found to depend on the instantaneous values of the boundary velocity and its time derivatives, again independent of past values. This situation is analogous to that encountered for finite-dimensional Hamiltonian systems with slowly-varying parameters, such as the pendulum with varying length. For this system, the adiabatic invariance of the action can be used to show that the amplitude of the motion depends only instantaneously on the various derivatives of the length.

To answer the second question, we use the first-order correction to the approximate velocity field obtained in (i). This $O(\varepsilon)$ accuracy in the velocity field is necessary to integrate the particle motion over the $O(1 / \varepsilon)$ timescale considered. Having computed the velocity field, the particle-position problem reduces to the solution of (independent) one-degree-of-freedom Hamiltonian systems with slowly-varying parameters. Since particles remain on vorticity contours, only the position along each contour, regarded as an angle variable, needs to be determined. The value of this angle is found to depend on the history of the boundary shape. It includes a geometric contribution, similar to the Hannay-Berry angle, which possesses a nice interpretation $[9,5,14,16,20]$. We note that the geometric angle has been studied in fluid dynamics in [22,23] where point-vortex solutions of the $2 \mathrm{~d}$ Euler equation are considered, and in [21] for Stokes flow; here it appears in the context of smooth inviscid flows.

In the following section, we present a short description of the $2 \mathrm{~d}$ Euler equation in a deforming domain in order to fix the notation, and we consider the behaviour of the leading-order Eulerian flow. The problem is formulated in terms of the diffeomorphism $g_{\Lambda}$, and the effect of infinitesimal domain deformations is described. Next, in Section 3 we compute the first-order correction to the Eulerian flow, which depends only on the instantaneous shape of the boundary and its velocity. Using these results, we study the Lagrangian flow in Section 4, where the geometric angle of the particle position is derived. In these sections, we consider general domains and arbitrary boundary deformations, requiring only that the boundary deformation be slow. The results are given as solutions of partial (pseudo)differential equations, which in general will have to be solved numerically. In Section 5 we develop a perturbative approach for the solution of these equations, based on the assumption of small (total) boundary deformation. We carry out the calculation to second order, but the Lie series formulation that we employ is well suited for systematic extensions to higher orders. An application to nearly axisymmetric flows in a slightly deformed disc is presented in Section 6, followed by a Discussion. Technical details and further developments are relegated to the Appendix.

As mentioned, it proves convenient to express our derivation in the language of differential forms in the space of the parameters defining the boundary shape. This makes explicit the linear dependence of several important quantities on the boundary velocity, and it gives a natural description of the geometric angle in terms of a curvature form in the parameter space. We use this language mainly as a notational tool, but it is clear that a more abstract geometric interpretation of the results could be given. The interested reader will find some details of this interpretation in the second part of the Discussion.

\section{Eulerian flow: Adiabatic invariance}

We begin by studying the behaviour of the Eulerian flow.

\subsection{Formulation}

Let $D_{\Lambda(\varepsilon t)} \subset \mathbb{R}^{2}$ be a simply-connected, bounded and smooth domain which is slowly evolving in time $t$ in a prescribed fashion while keeping its area fixed. Here $\Lambda$ denotes the set of parameters defining the shape of the boundary, and the slowness of their time 




Fig. 1. Parameterization of the shape of the domain $D_{\Lambda}$ by $\Lambda$ : as the parameter $\Lambda$ moves from $\Lambda(0)=0$ to $\Lambda(\varepsilon t)$ in $\mathcal{L}$, the fluid domain changes its shape from $D_{0}$ to $D_{\Lambda}$, inducing a change in the leading-order Eulerian flow whose streamlines are indicated.

dependence is made explicit by the introduction of the asymptotic parameter $\varepsilon \ll 1$. Denoting the (generally infinite-dimensional) space in which $\Lambda$ lives by $\mathcal{L}$, we can think of the evolution of the domain shape as the tracing of a curve $\Lambda(\varepsilon t) \subset \mathcal{L}$; see Fig. 1 . An example that can be kept in mind is the case where the domain boundary $\partial D_{\Lambda}$ can be described in polar coordinates $(r, \sigma)$ as a graph $r=r(\sigma ; \Lambda)$. When it is sufficiently smooth, this can be written as the Fourier series

$$
r(\sigma ; \Lambda)=\sum_{m=-\infty}^{\infty} \Lambda_{m} \mathrm{e}^{\mathrm{i} m \sigma},
$$

with $\Lambda_{-m}=\Lambda_{m}^{*}$, where $*$ denotes the complex conjugate. This gives an explicit form for the set of independent parameters $\Lambda=\left(\Lambda_{m}\right)_{m=0}^{\infty}$. As $D_{\Lambda}$ deforms, each of the parameters $\Lambda_{m}$ traces a curve in the complex plane, and the deformation is represented as a path in $\mathcal{L}=\mathbb{C}^{\mathbb{N}}$.

We can describe the evolution of a perfect fluid flow in $D_{\Lambda(\varepsilon t)}$ using the vorticity-streamfunction formulation

$$
\begin{aligned}
& \partial_{t} \omega+[\psi, \omega]=0, \\
& \omega=\Delta \psi .
\end{aligned}
$$

The velocity is given by $\mathbf{v}=(u, v)=\nabla^{\perp} \psi:=\left(-\partial_{y} \psi, \partial_{x} \psi\right), \omega=\nabla^{\perp} \cdot \mathbf{v}:=\partial_{x} v-\partial_{y} u$ is the vorticity, and $[f, g]:=\nabla^{\perp} f \cdot \nabla g=$ $\partial_{x} f \partial_{y} g-\partial_{x} g \partial_{y} f$ is the Jacobian. Steady flows satisfy $[\psi, \omega]=0$.

A convenient way of defining the domain boundary $\partial D_{\Lambda(\varepsilon t)}$ is as the level set $B(x, y ; \Lambda(\varepsilon t))=0$ of some prescribed function $B$. Since $\partial D_{\Lambda(\varepsilon t)}$ is a material curve,

$$
\partial_{t} B+[\psi, B]=0 \quad \text { for } B(\mathbf{x} ; \Lambda(\varepsilon t))=0 .
$$

Assuming that $\nabla B \neq 0$ on $\partial D_{\Lambda}$, this can be inverted to give the boundary condition

$$
\psi(\mathbf{x})=\varepsilon b(\mathbf{x} ; \varepsilon t) \quad \text { for } \mathbf{x} \in \partial D_{\Lambda(\varepsilon t)} .
$$

Here

$$
\varepsilon b(\mathbf{x} ; \varepsilon t)=\int_{\mathbf{x}_{0}}^{\mathbf{x}} \partial_{t} B\left(\mathbf{x}^{\prime} ; \Lambda(\varepsilon t)\right) \frac{\mathrm{d} l^{\prime}}{|\nabla B|},
$$

where the integral is taken along the boundary $\partial D_{\Lambda}$ and $\mathrm{d} l$ denotes arclength. (The fact that the area of $D_{\Lambda}$ is constant ensures that $b(\cdot ; \varepsilon t)$ is single valued.) We choose $\mathbf{x}_{0}$ in (2.6) such that

$$
\oint_{\partial D_{\Lambda(\varepsilon t)}} b(\varepsilon t) \mathrm{d} l=0 .
$$

It is clear from (2.5)-(2.6) that $\psi$ is linear in $\mathrm{d} \Lambda / \mathrm{d} t$ on $\partial D_{\Lambda}$.

In this paper, our concern is the behaviour of the flow for slow boundary deformations, which correspond formally to the limit $\varepsilon \rightarrow 0$. Long, $O\left(\varepsilon^{-1}\right)$ timescales are considered, so that $O(1)$ deformations are achieved. We make a blanket assumption that all functions are sufficiently smooth for our purposes, and we denote by $\mathcal{C}\left(D_{\Lambda}\right)$ the space of smooth real-valued functions in $D_{\Lambda}$. Exploiting the smallness of $\varepsilon$, we expand the vorticity and streamfunction in powers of $\varepsilon$ as

$$
\omega=\omega^{(0)}+\varepsilon \omega^{(1)}+\cdots \quad \text { and } \psi=\psi^{(0)}+\varepsilon \psi^{(1)}+\cdots
$$

Our aim in this section is to compute the leading-order flow $\psi^{(0)}$, given its initial value and the boundary deformation $b(\varepsilon t)$, and to show that it depends only on the boundary shape $\Lambda$ and not on its time history. 
First we note that the boundary conditions (2.5) imply that on $\partial D_{\Lambda}$,

$$
\psi^{(0)}=0 \quad \text { and } \quad \psi^{(1)}=b .
$$

Since the total vorticity $\omega$ is advected by the flow, the boundary $\partial D_{\Lambda}$ is a vorticity contour and thus on it we can take

$$
\omega=\omega^{(0)} \quad \text { and } \quad \omega^{(n)}=0 \text { for } n=1,2, \cdots
$$

on $\partial D_{\Lambda}$.

Substituting (2.8) into (2.2), we find

$$
\left[\psi^{(0)}, \omega^{(0)}\right]+\partial_{t} \omega^{(0)}+\varepsilon\left[\psi^{(1)}, \omega^{(0)}\right]+\varepsilon\left[\psi^{(0)}, \omega^{(1)}\right]+\varepsilon \partial_{t} \omega^{(1)}+O\left(\varepsilon^{2}\right)=0 .
$$

If the fluid flow is stable in the absence of boundary deformation, we expect that the flow will evolve only slowly when the boundary is deforming. We therefore introduce the slow time

$$
\tau=\varepsilon t,
$$

in terms of which (2.11) becomes

$$
\left[\psi^{(0)}, \omega^{(0)}\right]+\varepsilon \partial_{\tau} \omega^{(0)}+\varepsilon\left[\psi^{(1)}, \omega^{(0)}\right]+\varepsilon\left[\psi^{(0)}, \omega^{(1)}\right]+O\left(\varepsilon^{2}\right)=0 .
$$

At leading order we obtain

$$
\left[\psi^{(0)}, \omega^{(0)}\right]=0 .
$$

Taking into account the fact that $\psi^{(0)}=0$ on $\partial D$, we find that the leading-order flow $\psi^{(0)}$ is instantaneously steady. The relation (2.14) implies that there exists a scalar function $G$ relating $\omega^{(0)}$ and $\psi^{(0)}$,

$$
\psi^{(0)}=G\left(\omega^{(0)} ; \tau\right) .
$$

As noted, the function $G$ depends on the slow time $\tau$, regarded here as a parameter. We define $F$ as the inverse of $G$ : $G(F(u ; \tau) ; \tau)=u$ for every $u$ and $\tau$. With an abuse of notation, we will often write $G^{\prime}$ for $G^{\prime} \circ \omega^{(0)}=\nabla \psi^{(0)} / \nabla \omega^{(0)}$ and $F^{\prime}$ for $F^{\prime} \circ \psi^{(0)}=\nabla \omega^{(0)} / \nabla \psi^{(0)}$; what is meant will be clear from the context.

At $O(\varepsilon)$ we have

$$
\partial_{\tau} \omega^{(0)}+\left[\psi^{(1)}, \omega^{(0)}\right]+\left[\psi^{(0)}, \omega^{(1)}\right]=0 .
$$

Using (2.15), the second term can be written as

$$
\left[\psi^{(0)}, \omega^{(1)}\right]=G^{\prime}\left[\omega^{(0)}, \Delta \psi^{(1)}\right]=\left[\omega^{(0)}, G^{\prime} \Delta \psi^{(1)}\right],
$$

with which (2.16) becomes

$$
\begin{aligned}
& \partial_{\tau} \omega^{(0)}+\left[\phi, \omega^{(0)}\right]=0, \\
& \phi=\left[1-G^{\prime} \Delta\right] \psi^{(1)} .
\end{aligned}
$$

These two equations imply that the leading-order vorticity $\omega^{(0)}$ is rearranged by a divergence-free velocity field $\nabla^{\perp} \phi$ with $\phi$ related to the first-order streamfunction $\psi^{(1)}$ by (2.19). Note that (2.16)-(2.18) determine $\phi$ only up to the addition of a function of $\omega^{(0)}$. Our reason for making the particular choice (2.19) will be clear in Section 3.

\subsection{Determination of the Eulerian flow}

We now show how the leading-order flow $\psi^{(0)}$ can be determined from $(2.15)$ and the fact that $\omega^{(0)}(t)$ is a rearrangement of its initial value $\omega^{(0)}(0)$. We make the following two assumptions on $\psi^{(0)}$ :

H1. The leading-order streamfunction $\psi^{(0)}$ has only one critical point in $D_{\Lambda}$ (which is necessarily elliptic) and is nonlinearly stable in the sense of Arnold.

We recall that Arnold stability (see, e.g., $[4,10]$ ) requires that the steady streamfunction $\psi^{(0)}$ satisfies either (i) $0<c_{1} \leq G^{\prime} \leq$ $c_{2}<\infty$, or (ii) $0<1 / c_{\text {poi }}<c_{1} \leq-G^{\prime} \leq c_{2}<\infty$. In the second condition, $c_{\text {poi }}$ is the Poincaré constant for the domain $D_{\Lambda}$, namely the smallest eigenvalue $\mu$ of the problem

$$
(\Delta+\mu) u=0 \text { in } D_{\Lambda} \quad \text { with } \quad u=0 \text { on } \partial D_{\Lambda} .
$$


These conditions ensure that the steady flow is either a minimum or a maximum of the energy for fixed vorticity distribution. Note that $\mathrm{H} 1$ implies that

$$
-c_{\text {poi }}<F^{\prime}<\infty
$$

a condition which will be useful below. The assumption H1 is stronger than that made in WV but it considerably simplifies the solution of (2.43)-(2.45).

For the second assumption, we need a few more notation. Let $s$ denote a variable conjugate to $\psi^{(0)}$ in $D_{\Lambda}$, satisfying $\left[\psi^{(0)}, s\right]=1$. Denoting the differential arclength along the curve $\psi^{(0)}=$ const by $\mathrm{d} l$, we have $\mathrm{d} s=\mathrm{d} l /\left|\nabla \psi^{0}\right|$. We then assume:

H2. There exists a $c_{\psi}>0$ such that, for all values of c assumed by $\psi^{(0)}$,

$$
\oint_{\psi^{(0)}=c} \mathrm{~d} s \leq \frac{1}{c_{\psi}} .
$$

In the context of adiabatic invariance, this condition is natural: the left-hand side of (2.22) gives the period of rotation of fluid parcels along the streamline $\psi^{(0)}=c$; its boundedness ensures that a time-scale separation between this period and the timescale of the boundary deformation exists for sufficiently small $\varepsilon$. As noted in $\mathrm{WV}, \mathrm{H} 2$ holds if $\omega^{(0)} \neq 0$ at the fixed point of $\psi^{(0)}$.

For concreteness, we henceforth assume that, at $t=0$, our domain is parameterized by $\Lambda_{0}$ and we choose our coordinates in $\mathcal{L}$ such that $\Lambda_{0}=0$. Furthermore, we fix in $D_{\Lambda_{0}} \equiv D_{0}$ a steady leading-order flow $\psi^{(0)}(\mathbf{x}, 0)=\psi_{0}(\mathbf{x})$ satisfying H1-H2. Considering only the leading-order flow $\psi^{(0)}(\mathbf{x}, t)$ for the moment, we then claim that, assuming $\mathrm{H} 1-\mathrm{H} 2$ :

P1. The flow $\psi^{(0)}$ is uniquely determined by (i) the shape of the deformed domain $D_{\Lambda}$, (ii) the steadiness condition (2.15), and (iii) the fact that the vorticity $\omega^{(0)}=\Delta \psi^{(0)}$ is obtained by rearrangement of the initial vorticity $\omega_{0}=\Delta \psi_{0}$.

As a result, the leading-order flow at a fixed time $t$ depends only on the shape of the deformed domain at $t$ (parameterized by $\Lambda(\varepsilon t)$ ), and not on the history of shapes at intermediate times (parameterized by the path $\Lambda(\tau), 0<\tau<\varepsilon t$ ). One may draw an analogy with adiabatic invariance in finite-dimensional Hamiltonian systems with slowly-varying parameters such as the pendulum of varying length: here the amplitude of the oscillations is completely determined to leading order by the instantaneous value of the length, not by its time history.

To emphasize the fact that the leading-order flow depends on $\Lambda$ instantaneously, we introduce the notation

$$
\psi^{(0)}=\psi_{\Lambda} \quad \text { and } \quad \omega^{(0)}=\omega_{\Lambda}
$$

for the leading-order streamfunction and vorticity. We also write $G(\cdot ; \tau)=: G_{\Lambda(\tau)}(\cdot)$ and $F(\cdot ; \tau)=: F_{\Lambda(\tau)}(\cdot)$. These define the scalar functions $G_{\Lambda}$ and $F_{\Lambda}$, both of which have $\Lambda$ as a parameter. When no confusion may arise, we will write $G_{\Lambda} \circ \omega_{\Lambda}$ as $G_{\Lambda}$, $G_{\Lambda}^{\prime} \circ \omega_{\Lambda}$ as $G_{\Lambda}^{\prime}$, and similarly for $F_{\Lambda}$ and $F_{\Lambda}^{\prime}$.

We emphasize that the claim P1 is only local: it holds only for sufficiently small domain deformations. A similar result is proved in WV with a precise functional setting and a weaker set of hypotheses. The main idea, which we repeat here as parts of it are used throughout the paper, is to reformulate the problem in terms of the area-preserving diffeomorphism

$$
g_{\Lambda}: D_{0} \rightarrow D_{\Lambda}: \mathbf{x} \mapsto g_{\Lambda} \mathbf{x}
$$

which effects the vorticity rearrangement, that is, such that

$$
\omega_{\Lambda}=\omega_{0} \circ g_{\Lambda}^{-1}
$$

Since compositions of this type will appear frequently, it is convenient to introduce the pull-back notation and write this as

$$
\omega_{\Lambda}=\left(g_{\Lambda}^{-1}\right)^{*} \omega_{0} .
$$

Note that, for fixed $\omega_{0}$ and $\omega_{\Lambda}, g_{\Lambda}$ is not defined uniquely by (2.26): rearrangements along the lines of constant vorticity clearly have no effect. Correspondingly, the time derivative of $g_{\Lambda}$ is not necessarily the divergence-free velocity field $\nabla^{\perp} \phi$ appearing in (2.18)-(2.19), but the equality

$$
\frac{\mathrm{d}}{\mathrm{d} t} g_{\Lambda} \mathbf{x}=\nabla^{\perp}\left[\phi\left(g_{\Lambda} \mathbf{x} ; \Lambda\right)+\varpi\left(\omega_{\Lambda}\left(g_{\Lambda} \mathbf{x}\right)\right)\right]
$$

holds, where $\varpi$ is an arbitrary function of one variable. This non-uniqueness, of no importance as far as $\psi_{\Lambda}$ and $\omega_{\Lambda}$ are concerned, will play a crucial role when particle positions are examined in Section 4.

The map $g_{\Lambda}$ satisfies a nonlinear partial differential equation obtained as follows. Since $\omega_{\Lambda}$ is a steady flow in $D_{\Lambda}$, we have using (2.15),

$$
\omega_{\Lambda}=\Delta\left(G_{\Lambda} \circ \omega_{\Lambda}\right)
$$


so applying $g_{\Lambda}^{*}$, we find

$$
\omega_{0}=g_{\Lambda}^{*} \Delta\left(g_{\Lambda}^{-1}\right)^{*}\left(G_{\Lambda} \circ \omega_{0}\right) .
$$

The associated boundary conditions are

$$
g_{\Lambda}\left(\partial D_{0}\right)=\partial D_{\Lambda} \quad \text { and } \quad G_{\Lambda}\left(\omega_{\mathrm{b}}\right)=G_{0}\left(\omega_{\mathrm{b}}\right),
$$

where $\omega=\omega_{\mathrm{b}}$ on $\partial D_{\Lambda}$, which follows from (2.10) and the fact that $\psi_{\Lambda}=\psi_{0}=0$.

The partial differential equation (2.29), with $g_{\Lambda}$ and $G_{\Lambda}$ as unknowns, is exactly that obtained in WV in the context of the static problem. It involves the $g_{\Lambda}$-dependent operator $g_{\Lambda}^{*} \Delta\left(g_{\Lambda}^{-1}\right)^{*}$ which can be interpreted as the pull-back of the Laplacian $\Delta$ from the domain $D_{\Lambda}$ to the initial domain $D_{0}$ and is computed by expressing the partial derivatives $\partial_{\mathbf{x}}$ in terms of the transformed coordinates $\mathbf{X}=g_{\Lambda}^{-1} \mathbf{x}$. An explicit expression for (2.29) is given in WV (see (2.7)-(2.10) in that paper). There, this equation is shown to have a locally unique solution (modulo translations along vorticity contours) using a contraction mapping argument. This establishes P1 and provides a way of computing $g_{\Lambda}$ and $G_{\Lambda}$, and hence $\omega_{\Lambda}$ and $\psi_{\Lambda}$, in a manner that is clearly independent of the deformation history. Alternatively, P1 can be established using the stability assumption H1: the associated characterisation of steady flows as energy extrema makes it clear that the steady flow $\psi_{\Lambda}$ is the (locally unique) extremum in $D_{\Lambda}$ with vorticity distribution fixed by $\omega_{0}$.

We now consider the infinitesimal version of (2.29), that is, we consider the change in $g_{\Lambda}$ corresponding to an infinitesimal deformation of the domain. This yields a different construction for $g_{\Lambda}$, based on integration over $\Lambda$, and an alternative perspective on its path independence. Furthermore, the infinitesimal formulation provides all the ingredients needed for the computation of the first-order and higher-order corrections to $\psi_{\Lambda}$ and of the Lagrangian flow.

\subsection{Infinitesimal deformations}

In what follows, we will often make use of the fact that many important quantities are linear in the boundary deformation rate $\dot{\Lambda}:=\mathrm{d} \Lambda / \mathrm{d} \tau$. To factor out this dependence explicitly, we will regard these quantities as resulting from the pairing between vectors $\dot{\Lambda}$, which live in the tangent space $T_{\Lambda} \mathcal{L}$, and differential forms, which live in the dual space $T_{\Lambda}^{*} \mathcal{L}$. For instance, since the function $b$ in (2.6) is linear in $\dot{\Lambda}$, we can write it as

$$
b=\boldsymbol{\beta} \cdot \dot{\Lambda},
$$

where $\boldsymbol{\beta}$ is a one-form and denotes its pairing with the vector $\dot{\Lambda}$. By definition, $\boldsymbol{\beta}$ is a linear map from $T_{\Lambda} \mathcal{L}$ to the space $\mathcal{C}\left(\partial D_{\Lambda}\right)$ of real-valued functions defined on the boundary $\partial D_{\Lambda}$ :

$$
\boldsymbol{\beta}(\cdot ; \Lambda): T_{\Lambda} \mathcal{L} \rightarrow \mathcal{C}\left(\partial D_{\Lambda}\right) .
$$

With $\left(\Lambda_{m}\right)$ as coordinates in $\mathcal{L},\left(\partial / \partial \Lambda_{m}\right)$ as coordinates in $T_{\Lambda} \mathcal{L}$ and $\left(\mathbf{d} \Lambda_{m}\right)$ as coordinates in $T_{\Lambda}^{*} \mathcal{L}$, we can write

$$
\dot{\Lambda}=\dot{\Lambda}_{m} \partial / \partial \Lambda_{m}, \quad \boldsymbol{\beta}=\beta_{m} \mathbf{d} \Lambda_{m} \quad \text { and } \quad b=\beta_{m} \dot{\Lambda}_{m} .
$$

Here and henceforth, summation over repeated indices is implied. The $\Lambda_{m}$ are scalar coefficients while the $\beta_{m}$ are functions defined on $\partial D_{\Lambda}$. An explicit expression for $\beta_{m}$ is found directly from (2.6) as

$$
\beta_{m}=\int_{\mathbf{x}_{0}}^{\mathbf{x}} \frac{\partial}{\partial \Lambda_{m}} B\left(\mathbf{x}^{\prime} ; \Lambda\right) \frac{\mathrm{d} l^{\prime}}{|\nabla B|} .
$$

Working with differential forms like $\boldsymbol{\beta}$ gives a compact notation, factoring out the dependence on $\dot{\Lambda}$. At the same time, it allows for a geometric interpretation of our results as explained in the Discussion.

Let $\mathbf{d}$ be the exterior derivative in $\mathcal{L}$, so for any function (i.e. zero-form) $f$ of $\Lambda$, we have $\mathbf{d} f=\left(\partial f / \partial \Lambda_{m}\right) \mathbf{d} \Lambda_{m}$. Since

$$
\frac{\mathrm{d}}{\mathrm{d} \tau} g_{\Lambda}=\frac{\partial g_{\Lambda}}{\partial \Lambda_{m}} \dot{\Lambda}_{m}=\mathbf{d} g_{\Lambda} \cdot \dot{\Lambda}
$$

and $g_{\Lambda}$ is area preserving, we can define a one-form $\Phi$ with values in $\mathcal{C}\left(D_{\Lambda}\right)$ by

$$
\mathbf{d} g_{\Lambda}=\nabla^{\perp} \Phi \circ g_{\Lambda}
$$

The one-form $\Phi$ plays a central role in what follows; it encodes the manner in which an infinitesimal domain deformation (displacement in $\mathcal{L}$ ) induces a corresponding infinitesimal change of diffeomorphism $\mathbf{d} g_{\Lambda}$. The initial domain $\Lambda_{0} \equiv 0$ and initial flow $\psi_{0}$ having been fixed, $\Phi$ depends only on $\Lambda$ and takes its value in the space of functions in $D_{\Lambda}$; explicitly,

$$
\Phi(\cdot ; \Lambda): T_{\Lambda} \mathcal{L} \rightarrow \mathcal{C}\left(D_{\Lambda}\right)
$$


In the coordinates $\left(\mathbf{d} \Lambda_{m}\right)$ in $T_{\Lambda}^{*} \mathcal{L}, \Phi$ takes the more explicit form $\Phi=\Phi_{m} \mathbf{d} \Lambda_{m}$, where the $\Phi_{m} \in \mathcal{C}\left(D_{\Lambda}\right)$ satisfy

$$
\frac{\partial g_{\Lambda}}{\partial \Lambda_{m}}=\nabla^{\perp} \Phi_{m} \circ g_{\Lambda}
$$

Using the correspondence between functions on $D_{\Lambda}$ and divergence-free vector fields afforded by the operator $\nabla^{\perp}, \Phi$ can be identified with a vector(-field)-valued one-form. Differential forms of this type arise in many contexts in geometry, where they define connections (see, e.g., [7, ch. 18] and [20]); we shall henceforth refer to $\Phi$ as a connection, leaving the more detailed geometric interpretation to the Discussion.

Taking the exterior derivative of (2.26) and using the definition (2.36) gives

$$
\mathbf{d} \omega_{\Lambda}+\left[\Phi, \omega_{\Lambda}\right]=0,
$$

which reads in components,

$$
\frac{\partial \omega_{\Lambda}}{\partial \Lambda_{m}}+\left[\Phi_{m}, \omega_{\Lambda}\right]=0
$$

Pairing (2.39) with $\dot{\Lambda}$, we recover

$$
\partial_{\tau} \omega_{\Lambda}+\left[\Phi \cdot \dot{\Lambda}, \omega_{\Lambda}\right]=0,
$$

which is equivalent to (2.18)— the relationship between $\Phi \cdot \dot{\Lambda}$ and $\phi$ will be made clear in Section 3 below. Unlike $\omega_{\Lambda}$, the leadingorder streamfunction $\psi_{\Lambda}$ is not simply rearranged as $\Lambda$ changes. Applying $\mathbf{d}$ to $\psi_{\Lambda}=G_{\Lambda}\left(\omega_{\Lambda}\right)$ and using (2.39) show that

$$
\mathbf{d} \psi_{\Lambda}+\left[\Phi, \psi_{\Lambda}\right]=\mathbf{d} G_{\Lambda} \circ \omega_{\Lambda} .
$$

Here and elsewhere, in $\mathbf{d} G_{\Lambda} \circ \omega_{\Lambda}$ the exterior derivative is taken with respect to the (parametric) dependence of $G_{\Lambda}$ on $\Lambda$, so $\mathbf{d}\left(G_{\Lambda} \circ \omega_{\Lambda}\right)=\mathbf{d} G_{\Lambda} \circ \omega_{\Lambda}+\left(G_{\Lambda}^{\prime} \circ \omega_{\Lambda}\right) \mathbf{d} \omega_{\Lambda}$.

We next obtain a dynamical equation for $\Phi$. Taking the derivative $\mathbf{d}$ of (2.29) and after a little algebra, we can write the result in the form

$$
\left(\Delta-F_{\Lambda}^{\prime}\right)\left[\Phi, \psi_{\Lambda}\right]-\Delta\left(\mathbf{d} G_{\Lambda} \circ \omega_{\Lambda}\right)=0 .
$$

The corresponding boundary conditions are

$$
\Phi=\boldsymbol{\beta} \text { on } \partial D_{\Lambda},
$$

which follows from (2.5), (2.31) and (2.19), taking into account that

$$
\mathbf{d} G_{\Lambda} \circ \omega_{\Lambda}=0 \text { on } \partial D_{\Lambda},
$$

which follows from the fact $\psi_{\Lambda}=0$ on $\partial D_{\Lambda}$. The system (2.43)-(2.45), which is the infinitesimal version of (2.29), is central to this paper. Its solution tells us how a steady flow $\omega_{\Lambda}$ changes as a result of boundary deformation $\dot{\Lambda}$ through the infinitesimal rearrangement $\left[\Phi \cdot \dot{\Lambda}, \omega_{\Lambda}\right]$. As we shall see shortly, the term $\Delta\left(\mathbf{d} G_{\Lambda} \circ \omega_{\Lambda}\right)$ can be regarded as arising from a constraint. In components, (2.43) takes the form

$$
\left(\Delta-F_{\Lambda}^{\prime}\right)\left[\Phi_{m}, \psi_{\Lambda}\right]-\Delta\left(\left(\partial G_{\Lambda} / \partial \Lambda_{m}\right) \circ \omega_{\Lambda}\right)=0
$$

with boundary conditions

$$
\Phi_{m}=\boldsymbol{\beta}_{m} \quad \text { and } \quad\left(\partial G_{\Lambda} / \partial \Lambda_{m}\right) \circ \omega_{\Lambda}=0 .
$$

We can solve (2.43)-(2.45) for $\left[\Phi, \psi_{\Lambda}\right]$ and $\mathbf{d} G_{\Lambda}$ as follows. For any function $u \in \mathcal{C}\left(D_{\Lambda}\right)$, we define $\mathrm{P}_{\Lambda} u$ as the part of $u$ with zero mean along streamlines. Explicitly,

$$
\left(\mathrm{P}_{\Lambda} u\right)(\mathbf{x}):=u(\mathbf{x})-\left\{\oint_{\psi_{\Lambda}=\psi_{\Lambda}(\mathbf{x})} u \mathrm{~d} s\right\} /\left\{\oint_{\psi_{\Lambda}=\psi_{\Lambda}(\mathbf{x})} \mathrm{d} s\right\},
$$

where, as before, $\mathrm{d} s=\mathrm{d} l /\left|\nabla \psi_{\Lambda}\right|$. It follows from this definition that if $u$ is constant on a contour of constant $\psi_{\Lambda}, \mathrm{P}_{\Lambda} u=0$. Note that since the contours of $\omega_{\Lambda}$ and $\psi_{\Lambda}$ coincide, an equivalent definition of $\mathrm{P}_{\Lambda}$ could have been given in terms of integrals along vorticity contours $\omega_{\Lambda}=c$, but this is less convenient since we may have $\nabla \omega_{\Lambda}=0$ while $\nabla \psi_{\Lambda} \neq 0$ by hypothesis. By $\mathrm{H} 2$, the denominator in (2.48) is never zero, so $\mathrm{P}_{\Lambda} u$ is smooth if $u$ is. Letting $\varphi=\left[\psi_{\Lambda}, \Phi\right]-\mathbf{d} G_{\Lambda} \circ \omega_{\Lambda}$, and using the facts that $\mathrm{P}_{\Lambda}\left[\psi_{\Lambda}, \Phi\right]=\left[\psi_{\Lambda}, \Phi\right]$ and $\mathrm{P}_{\Lambda}\left(\mathbf{d} G_{\Lambda} \circ \omega_{\Lambda}\right)=0$, we then have

$$
\left[\psi_{\Lambda}, \Phi\right]=\mathrm{P}_{\Lambda} \varphi \quad \text { and } \quad \mathbf{d} G_{\Lambda} \circ \omega_{\Lambda}=\left(1-\mathrm{P}_{\Lambda}\right) \varphi
$$


Hence we can write (2.43) as

$$
\left(\Delta-F_{\Lambda}^{\prime} \mathrm{P}_{\Lambda}\right) \varphi=0,
$$

which is a linear pseudodifferential equation involving $\varphi$ only. Following (2.44) and (2.45), the boundary conditions for $\varphi$ are

$$
\varphi=\left[\psi_{\Lambda}, \boldsymbol{\beta}\right] \text { on } \partial D_{\Lambda}
$$

It is shown in Appendix A that (2.50)-(2.51) can be solved uniquely for $\varphi$. Using (2.49), we recover $\left[\psi_{\Lambda}, \Phi\right]$ and $\mathbf{d} G_{\Lambda}$.

From $\left[\psi_{\Lambda}, \Phi\right], \Phi$ can be solved up to the addition of a one-form $\Pi \circ \omega_{\Lambda}$, which depends on space through $\omega_{\Lambda}$ only; here $\Pi$ is an arbitrary one-form whose values $\Pi \cdot \dot{\Lambda}$ are real functions of a single variable. The zero-mean part $\mathrm{P}_{\Lambda} \Phi$, on the other hand, can be solved from $\left[\psi_{\Lambda}, \Phi\right]$ in a unique fashion. We can therefore write

$$
\Phi=\mathrm{P}_{\Lambda} \Phi+\Pi \circ \omega_{\Lambda}
$$

where $\Pi$ is arbitrary. The non-uniqueness of $\Phi$ simply reflects at the infinitesimal level the non-uniqueness of $g_{\Lambda}$, which is only defined up to displacements along contours of $\omega_{\Lambda}$. The freedom in choosing a particular function $\Pi$ can be thought of as a gauge freedom in the definition of $\Phi$. A natural choice for the gauge $\Pi$ will appear in the next section.

Once we solve the linear problem (2.43), the solution of the nonlinear problem (2.29) for $g_{\Lambda}$ can be obtained by integration (or by the iterative approach in WV), at least in a neighbourhood of $\Lambda=0$ and subject to sufficient smoothness of the flow and the domain. Given a sequence of boundary deformation, that is, given a path $\tau \mapsto \Lambda(\tau) \in \mathcal{L}$, one can in principle solve (2.43) and find $\Phi$ for each $\Lambda$ as long as the flows $\psi_{\Lambda}$ encountered along the path satisfy $\mathrm{H} 1-\mathrm{H} 2$.

We conclude this section by discussing how the fact that $g_{\Lambda}$ is independent of the path in $\mathcal{L}$ (modulo translations along contours of $\omega_{\Lambda}$ ) is reflected at the infinitesimal level. Assuming that the connection form $\Phi$ is known, we consider the non-integrability of the diffeomorphism $g_{\Lambda}$ that $\Phi$ generates. In other words, we consider two distinct paths $\gamma_{1}$ and $\gamma_{2}$, both connecting the same two points in parameter space $\mathcal{L}$, and examine how the two diffeomorphisms $g_{\gamma_{1}}$ and $g_{\gamma_{2}}$, generated along these paths, differ. By taking the two paths to be infinitesimal, it can be shown as detailed in Appendix B.1 that the non-integrability is encoded in the curvature of $\Phi$. This is given by

$$
\kappa:=\mathbf{d} \Phi+\frac{1}{2}[\Phi \wedge \Phi]
$$

where the bracket $[\cdot \wedge \cdot]$ is defined in coordinates by

$$
[\alpha \wedge \eta]=\left[\alpha_{m}, \eta_{n}\right] \mathbf{d} \Lambda_{m} \wedge \mathbf{d} \Lambda_{n}
$$

for any two one-forms $\alpha=\alpha_{m} \mathbf{d} \Lambda_{m}$ and $\eta=\eta_{n} \mathbf{d} \Lambda_{n}$ with values in $\mathcal{C}\left(D_{\Lambda}\right)$. (Note that in contrast with $[f, f]=0$ for any function $f,[\alpha \wedge \alpha] \neq 0$ in general.) The curvature $\kappa$ is a two-form over the parameter space $\mathcal{L}$ with values in $\mathcal{C}\left(D_{\Lambda}\right)$; it is expressed in coordinates as $\kappa=\kappa_{m n} \mathbf{d} \Lambda_{m} \wedge \mathbf{d} \Lambda_{n}$, where $\kappa_{m n}=-\kappa_{n m}$. Its integral over the area enclosed by the curves $\gamma_{1}$ and $\gamma_{2}$ is the diffeomorphism $g_{\gamma_{1}} g_{\gamma_{2}}^{-1}$ which quantifies the difference between $g_{\gamma_{1}}$ and $g_{\gamma_{2}}$.

In Appendix B.2, we deduce from (2.39) that

$$
\left[\kappa, \omega_{\Lambda}\right]=0 .
$$

This is the infinitesimal expression of the independence of $\omega_{\Lambda}$ on the deformation history. Eq. (2.55) implies that the curvature $\kappa$ depends on $\mathbf{x}$ only through the vorticity $\omega_{\Lambda}$ : explicitly,

$$
\kappa=w \circ \omega_{\Lambda}
$$

for some two-form $w$ whose values are functions of a single variable. This integrability condition, an infinitesimal version of the statement that $g_{\Lambda}$ is a unique function of $\Lambda$ modulo displacements along lines of constant $\omega_{\Lambda}$, turns out to be important when we consider the Lagrangian particle trajectories in Section 4. Note that (2.55) is consistent with the dynamical equation (2.43) for $\Phi$ in the sense that it can also be derived directly from (2.43). This is verified in Appendix B.3.

\section{Eulerian flow: First-order correction to the streamfunction}

We now turn to the derivation of the first-order correction $\psi^{(1)}$ to the leading-order flow $\psi_{\Lambda}$. This is needed to determine the trajectories of fluid particles over the $O\left(\varepsilon^{-1}\right)$ timescales of interest. Remarkably, $\psi^{(1)}$ can be derived from the knowledge of $\Phi$ alone. In the process, the gauge of $\Phi$ is fixed in what we argue is a natural manner. Higher-order corrections can also be obtained through a systematic iterative procedure which we set out in Appendix $\mathrm{C}$; there we show that these corrections are local in $\Lambda$ in the sense that they depend only on $\dot{\Lambda}, \ddot{A}$, etc. and not on the history of the domain deformation. 
We start by deriving an integral constraint imposed by the material conservation of vorticity. Since $\omega_{\Lambda}$ and $\omega=\omega_{\Lambda}+\varepsilon \omega^{(1)}+\cdots$ are both rearrangements of the initial vorticity $\omega_{0}$, the areas inside the contours of $\omega_{\Lambda}$ and $\omega$ are the same, namely,

$$
\int_{D} \Theta\left(\omega_{\Lambda}+\varepsilon \omega^{(1)}+\cdots-\Omega\right) \mathrm{d}^{2} \mathbf{x}=\int_{D} \Theta\left(\omega_{\Lambda}-\Omega\right) \mathrm{d}^{2} \mathbf{x}
$$

for any $\Omega$ in the range of values taken by $\Omega_{\Lambda}$. Here $\Theta(s)=1$ if $s \geq 0$ and $\Theta(s)=0$ otherwise is the Heaviside function. Expanding in $\varepsilon$ gives to leading order

$$
\int_{D} \delta\left(\omega_{\Lambda}-\Omega\right) \omega^{(1)} \mathrm{d}^{2} \mathbf{x}=\oint \omega^{(1)} \frac{\mathrm{d} l}{\left|\nabla \omega_{\Lambda}\right|}=G_{\Lambda}^{\prime} \oint \omega^{(1)} \mathrm{d} s=0 .
$$

In terms of the projection $\mathrm{P}_{\Lambda}$, this constraint on $\omega^{(1)}$ can be written as

$$
\left(1-\mathrm{P}_{\Lambda}\right) \omega^{(1)}=\left(1-\mathrm{P}_{\Lambda}\right) \Delta \psi^{(1)}=0 .
$$

Turning now to the computation of $\psi^{(1)}$, we note that (2.27) and (2.36) imply that $\phi$ and $\Phi \cdot \dot{\Lambda}$ differ by a function of $\omega_{\Lambda}$ only. Thus we can write

$$
\phi=\Phi^{\star} \cdot \dot{\Lambda},
$$

where

$$
\Phi^{\star}=\mathrm{P}_{\Lambda} \Phi+\Pi^{\star} \circ \omega_{\Lambda}
$$

Here $\Phi^{\star}$ is a particular choice of the connection $\Phi$ which corresponds to the unique choice of the gauge $\Pi=\Pi^{\star}$ in $(2.52)$ that ensures that (3.4) holds. In this sense $\Phi^{\star}$ can be seen as a natural choice of the connection $\Phi$. The computation which follows shows how it can be obtained from $\mathrm{P}_{\Lambda} \Phi$.

Since $\psi^{(1)}$, like $\phi$, is linear in $\dot{\Lambda}$, we can write

$$
\psi^{(1)}=\Psi^{(1)} \cdot \dot{\Lambda}
$$

where, $\Psi^{(1)}$, like $\Phi$, is a function-valued form; their relationship follows from (2.19) as

$$
\left(1-G_{\Lambda}^{\prime} \Delta\right) \Psi^{(1)}=\mathrm{P}_{\Lambda} \Phi+\Pi^{\star} \circ \omega_{\Lambda} .
$$

Applying $F_{\Lambda}^{\prime} \mathrm{P}_{\Lambda}$ to this and using (3.3), we find

$$
\left(\Delta-F_{\Lambda}^{\prime} \mathrm{P}_{\Lambda}\right) \Psi^{(1)}=-F_{\Lambda}^{\prime} \mathrm{P}_{\Lambda} \Phi,
$$

which, along with the associated boundary condition [cf. (2.9)]

$$
\Psi^{(1)}=\boldsymbol{\beta} \quad \text { on } \partial D_{\Lambda},
$$

determines $\Psi^{(1)}$ uniquely. More explicitly, (3.8) is well posed, with the right-hand side that is uniquely defined in spite of the gauge freedom in $\Phi$. The operator on the left-hand side is the same as that in (2.50) and hence its invertibility can be established using the same arguments, detailed in Appendix A.

Applying $\left(1-\mathrm{P}_{\Lambda}\right)$ to $(3.7)$, we find

$$
\Pi^{\star} \circ \omega_{\Lambda}=\left(1-\mathrm{P}_{\Lambda}\right) \Psi^{(1)} .
$$

This gives us $\Pi^{\star}$, and thus the natural connection $\Phi^{\star}$, once $\Psi^{(1)}$ is determined from (3.8). Note that (3.5) and (3.10) imply that it satisfies

$$
\left(1-\mathrm{P}_{\Lambda}\right) \Psi^{(1)}=\left(1-\mathrm{P}_{\Lambda}\right) \Phi^{\star} \Leftrightarrow \oint_{\psi_{\Lambda}=c} \Psi^{(1)} \mathrm{d} s=\oint_{\psi_{\Lambda}=c} \Phi^{\star} \mathrm{d} s .
$$

This relation turns out to be crucial for the computation of fluid particle trajectories in the next section.

\section{Lagrangian flow: Geometric angle}

In this section we study the evolution of fluid (or tracer) particles in our flow over a timescale $\tau=O(1)$. 


\subsection{Hamiltonian formulation}

Up to this point, our description of the Eulerian dynamics has been (mostly) coordinate independent. But in order to study particle positions, we need to introduce explicit coordinates $(x, y)$ in $D_{\Lambda} ;(x, y)$ is chosen to coincide with the fixed coordinates in the ambient space $\mathbb{R}^{2}$ through which $D_{\Lambda(\varepsilon t)}$ moves.

The evolution of a particle with position $(x(t), y(t))$ moving with the fluid is governed by the Hamiltonian system

$$
\frac{\mathrm{d} x}{\mathrm{~d} t}=-\frac{\partial \psi}{\partial y} \quad \text { and } \quad \frac{\mathrm{d} y}{\mathrm{~d} t}=\frac{\partial \psi}{\partial x},
$$

with the streamfunction $\psi$ acting as the Hamiltonian. Our aim here is to obtain an estimate of $(x(t), y(t))$ with an error of $O(\varepsilon)$ for $\tau=O(1)$, so in the rest of this section we put

$$
H(x, y, t)=\psi_{\Lambda(\varepsilon t)}(x, y)+\varepsilon \Psi^{(1)}(x, y ; \Lambda(\varepsilon t)) \cdot \dot{\Lambda}
$$

in place of $\psi$ in (4.1), keeping in mind the validity of this approximation.

For $\varepsilon=0$ and hence $\Lambda$ constant, the Hamiltonian (4.2) is integrable. For $\varepsilon \neq 0$, two types of perturbations make it nonintegrable: the slow time dependence of $\psi_{\Lambda}$ introduced by the time dependence of $\Lambda$, and the $O(\varepsilon)$ change introduced by the addition of $\psi^{(1)}=\Psi^{(1)} \cdot \dot{\Lambda}$. We examine the combined effect of these two perturbations following closely the approach of [5].

Since the leading-order Hamiltonian $\psi_{\Lambda}$ is integrable for fixed $\Lambda$, we first change to action-angle variables (e.g., [3, p. 297ff]). At each $(x, y)$, we define the action $I$ by

$$
I(x, y)=\frac{1}{2 \pi} \int_{\operatorname{int}\left\{\psi_{\Lambda}=\psi_{\Lambda}(x, y)\right\}} \mathrm{d}^{2} \mathbf{x}=: \frac{1}{2 \pi} A\left(\psi_{\Lambda}\right) .
$$

The angle $\theta$ is defined as the variable conjugate to $I,[I, \theta]=1$. It is $2 \pi$-periodic since the contours of $\psi_{\Lambda}$ are closed and it is related to the variable $s$ used earlier by

$$
2 \pi \mathrm{d} s=A^{\prime}\left(\psi_{\Lambda}\right) \mathrm{d} \theta
$$

The canonical transformation $(x, y) \mapsto(I, \theta)$ is obtained by a generating function $S(I, y ; \Lambda)$, with

$$
x=\frac{\partial S}{\partial y} \quad \text { and } \quad \theta=\frac{\partial S}{\partial I}
$$

Solving these implicit equations, we can write

$$
x=X(I, \theta ; \Lambda) \text { and } y=Y(I, \theta ; \Lambda) .
$$

With these and (4.3), we define

$$
\begin{aligned}
& \hat{\psi}_{\Lambda}(I ; \Lambda)=\psi^{(0)}(X(I, \theta ; \Lambda), Y(I, \theta ; \Lambda) ; \Lambda)=A^{-1}(2 \pi I ; \Lambda), \\
& \hat{\Psi}^{(1)}(I, \theta ; \Lambda)=\Psi^{(1)}(X(I, \theta ; \Lambda), Y(I, \theta ; \Lambda) ; \Lambda) .
\end{aligned}
$$

Here and in the rest of this section, we denote by a hât quantities considered as functions of $(I, \theta)$, except for $\hat{H}$ defined in $(4.9)$.

So far we have considered a fixed value of $\Lambda$. Now let $\Lambda$ evolve slowly in time, $\Lambda=\Lambda(\varepsilon t)$. The equations of motion in $(I, \theta)$ variables are

$$
\frac{\mathrm{d} I}{\mathrm{~d} t}=-\frac{\partial \hat{H}}{\partial \theta} \quad \text { and } \quad \frac{\mathrm{d} \theta}{\mathrm{d} t}=\frac{\partial \hat{H}}{\partial I},
$$

where the new Hamiltonian $\hat{H}(I, \theta ; \Lambda)$ is related to $H(x, y ; \Lambda)$ by

$$
\hat{H}(I, \theta ; \Lambda)=H(X(I, \theta ; \Lambda), Y(I, \theta ; \Lambda) ; \Lambda)+\frac{\partial S}{\partial t} .
$$

We note an abuse of notation here: properly speaking $H=H(x, y, t)$, but since the $t$-dependence only enters through $\Lambda(\varepsilon t)$ and its derivative, we have written $H=H(x, y ; \Lambda(\varepsilon t))$. Differentiating the definition

$$
\hat{S}(I, \theta ; \Lambda)=S(I, Y(I, \theta ; \Lambda) ; \Lambda)
$$

with respect to $t$ at fixed $(I, \theta)$ gives

$$
\mathbf{d} \hat{S} \cdot \frac{\mathrm{d} \Lambda}{\mathrm{d} t}=\frac{\partial S}{\partial t}+\frac{\partial S}{\partial y} \frac{\partial Y}{\partial t}=\frac{\partial S}{\partial t}+X \mathbf{d} Y \cdot \frac{\mathrm{d} \Lambda}{\mathrm{d} t} .
$$


Upon substituting $\partial S / \partial t$ into (4.9), we obtain

$$
\hat{H}(I, \theta ; \Lambda)=\hat{\psi}_{\Lambda}(I)+\varepsilon \hat{\Psi}^{(1)}(I, \theta ; \Lambda) \cdot \dot{\Lambda}+\varepsilon\{\mathbf{d} \hat{S}(I, \theta ; \Lambda)-X(I, \theta ; \Lambda) \mathbf{d} Y(I, \theta ; \Lambda)\} \cdot \dot{\Lambda}
$$

Since the particles are attached to contours of vorticity $\omega=$ const, which only deviate by $O(\varepsilon)$ from the corresponding contours of $\omega_{\Lambda}$, the action can only vary by $O(\varepsilon)$ over timescales $\tau=O(1)$. This is also evident from direct computation: since $\hat{H}$ is independent of $\theta$ at leading order and is periodic in $\theta$ at the next order,

$$
\frac{\mathrm{d} I}{\mathrm{~d} t}=-\varepsilon \frac{\partial}{\partial \theta}\left\{\hat{\Psi}^{(1)}+\mathbf{d} \hat{S}-X \mathbf{d} Y\right\} \cdot \dot{\Lambda}
$$

and the principle of averaging (e.g., [3, Section 52]) implies that $I$ changes only by $O(\varepsilon)$ for $\tau=O(1)$.

The behaviour of the angle variable is more interesting. From (4.8) we have

$$
\frac{\mathrm{d} \theta}{\mathrm{d} t}=\frac{\partial \hat{\psi}_{\Lambda}}{\partial I}+\varepsilon \frac{\partial}{\partial I}\left\{\hat{\Psi}^{(1)}+\mathbf{d} \hat{S}-X \mathbf{d} Y\right\} \cdot \dot{\Lambda} .
$$

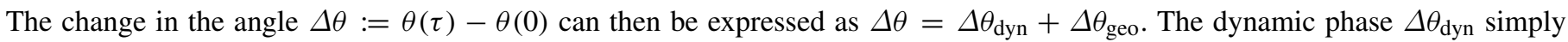
arises from the instantaneous frequency of the particle, which is the first term in (4.14) above,

$$
\Delta \theta_{\mathrm{dyn}}=\frac{1}{\varepsilon} \frac{\partial}{\partial I} \int_{0}^{\tau} \hat{\psi}_{\Lambda\left(\tau^{\prime}\right)}(I) \mathrm{d} \tau^{\prime}
$$

The other terms make up the geometric angle $\Delta \theta_{\text {geo }}$.

\subsection{Geometric angle $\Delta \theta_{\text {geo }}$}

In this subsection we show that, as in the finite-dimensional cases of [9,5], the angle $\Delta \theta_{\text {geo }}$ can be understood in geometric terms as the (an)holonomy of a connection as a closed path is traversed in a parameter space.

From (4.14), the geometric angle $\Delta \theta_{\text {geo }}$ can be written as

$$
\Delta \theta_{\text {geo }}=\int_{0}^{\tau} \frac{\partial}{\partial I}\left\{\hat{\Psi}^{(1)}\left(I, \theta ; \Lambda\left(\tau^{\prime}\right)\right)+\mathbf{d} S\left(I, \theta ; \Lambda\left(\tau^{\prime}\right)\right)-X\left(I, \theta ; \Lambda\left(\tau^{\prime}\right)\right) \mathbf{d} Y\left(I, \theta ; \Lambda\left(\tau^{\prime}\right)\right)\right\} \cdot \frac{\mathrm{d} \Lambda}{\mathrm{d} \tau^{\prime}} \mathrm{d} \tau^{\prime} .
$$

This form suggests that $\Delta \theta_{\text {geo }}$ depends only on the path traversed in $\mathcal{L}$ and not on its time parameterization. The terms inside the braces do depend on $I$ and $\theta$, but as shown earlier, the total variation of the action $I$ is of $O(\varepsilon)$ over the timescale of interest. The dependence on the periodic variable $\theta$ can be removed by averaging. For any function $f$ periodic in $\theta$, let

$$
\langle f\rangle:=\frac{1}{2 \pi} \int_{0}^{2 \pi} f(\theta) \mathrm{d} \theta .
$$

We note that by (4.4) this is essentially equivalent to the projection $1-\mathrm{P}_{\Lambda}$. Applying $\langle\cdot\rangle$ to $(4.16)$ and replacing $I(t)$ by $I(0)$, we find

$$
\Delta \theta_{\mathrm{geo}}=\frac{\partial}{\partial I} \int_{C_{\Lambda}}\left\langle\hat{\Psi}^{(1)}+\mathbf{d} S-X \mathbf{d} Y\right\rangle+O(\varepsilon),
$$

where $C_{\Lambda}$ is the path traversed in $\mathcal{L}$ and where the integrand depends only on $I$ and $\Lambda$.

Due to the arbitrariness in the angle coordinates (depending on our choice of $\theta=0$ for each $\Lambda$ ), the geometric angle is only unambiguously defined when the path $C_{\Lambda}$ is closed, that is, when $\Lambda(\tau)=\Lambda(0)=0$. Following $[9,5]$, we consider this scenario, which is illustrated in Fig. 2. Since $\mathbf{d} S$ is exact, it vanishes when integrated around $C_{\Lambda}$. Using Stokes' theorem in $\mathcal{L}$, the remaining terms in (4.18) can be written as

$$
\Delta \theta_{\text {geo }}=\frac{\partial}{\partial I} \int_{\mathcal{S}_{\Lambda}}\left\langle\mathbf{d} \hat{\Psi}^{(1)}-\mathbf{d} X \wedge \mathbf{d} Y\right\rangle,
$$

where $\mathcal{S}_{\Lambda}$ is a $2 \mathrm{~d}$ surface bounded by $C_{\Lambda}$. The second term is identical to that obtained in [5, Eq. (18)] for general Hamiltonian systems depending slowly on time; the first term results from the $O(\varepsilon)$ change to the Hamiltonian induced by the boundary deformation.

Now $\left\langle\hat{\Psi}^{(1)}\right\rangle=\left\langle\hat{\Phi}^{\star}\right\rangle$ by (3.11), so the first term in the integral can be written as

$$
\left\langle\mathbf{d} \hat{\Psi}^{(1)}\right\rangle=\left\langle\mathbf{d} \hat{\Phi}^{\star}\right\rangle .
$$




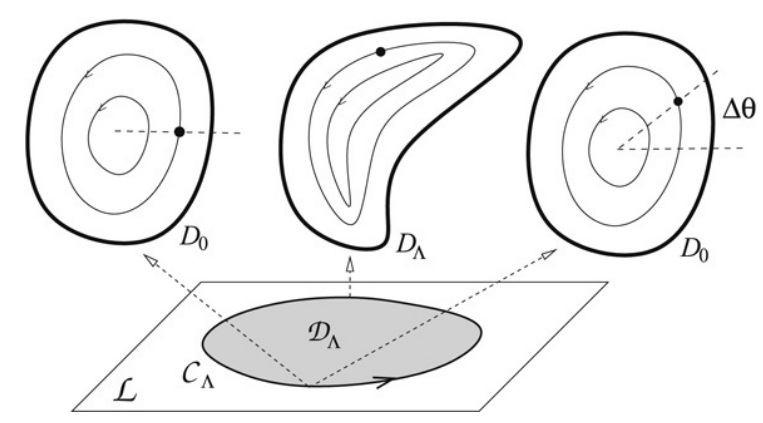

Fig. 2. Angle change for a cyclic domain deformation. As $\Lambda(\tau)$ describes the closed loop $\mathcal{C}_{\Lambda} \subset \mathcal{L}$, with interior $\mathcal{D}_{\Lambda}$, the fluid domain $D_{\Lambda}$ is deformed and returns to its original shape. Fluid particles remain on vorticity contours which approximately coincide with streamlines. The position of the particles along vorticity contours is defined by the angle-like variable $\theta$ whose total change $\Delta \theta$ includes the geometric contribution $\Delta \theta_{\text {geo }}$ which depends only on the geometrical properties of $\mathcal{D}_{\Lambda}$.

As for the second term, we use the fact that $g_{\Lambda}$ is a canonical transformation (since it is area preserving) to write

$$
\mathbf{X}(I, \theta ; \Lambda)=g_{\Lambda} \mathbf{X}(I, \theta ; 0),
$$

thus defining the transformation to action-angle coordinates for all values of $\Lambda$ in terms of the transformation at $\Lambda=0$.

Returning to (4.21), it follows from this and (2.36) that

$$
\mathbf{d X}(I, \theta ; \Lambda)=\left.\nabla^{\perp} \Phi\right|_{\mathbf{X}(I, \theta ; \Lambda)} .
$$

We then have

$$
\begin{aligned}
\mathbf{d} X \wedge \mathbf{d} Y & =\frac{\partial X}{\partial \Lambda_{m}} \frac{\partial Y}{\partial \Lambda_{n}} \mathbf{d} \Lambda_{m} \wedge \mathbf{d} \Lambda_{n}=-\frac{\partial \Phi_{m}}{\partial Y} \frac{\partial \Phi_{n}}{\partial X} \mathbf{d} \Lambda_{m} \wedge \mathbf{d} \Lambda_{n} \\
& =\frac{1}{2}\left[\Phi_{m}, \Phi_{n}\right] \mathbf{d} \Lambda_{m} \wedge \mathbf{d} \Lambda_{n}=\frac{1}{2}[\Phi \wedge \Phi]=\frac{1}{2}\left[\Phi^{\star} \wedge \Phi^{\star}\right]
\end{aligned}
$$

where the last equality follows from the fact that the bracket is independent of the gauge choice for $\Phi$. Furthermore, the fact that the transformation to action-angle variables is canonical implies that

$$
\frac{1}{2}\left[\Phi^{\star} \wedge \Phi^{\star}\right]=\frac{1}{2}\left[\hat{\Phi}^{\star} \wedge \hat{\Phi}^{\star}\right],
$$

where the second bracket is in terms of $(I, \theta)$. We can therefore write (4.19) as

$$
\Delta \theta_{\text {geo }}=\frac{\mathrm{d}}{\mathrm{d} I} \int_{\mathcal{S}_{\Lambda}}\left\langle\mathbf{d} \hat{\Phi}^{\star}-\frac{1}{2}\left[\hat{\Phi}^{\star} \wedge \hat{\Phi}^{\star}\right]\right\rangle .
$$

A last step, detailed in Appendix B.4, shows that

$$
\mathbf{d} \hat{\Phi}^{\star}-\frac{1}{2}\left[\hat{\Phi}^{\star} \wedge \hat{\Phi}^{\star}\right]=\mathbf{d} \Phi^{\star}+\frac{1}{2}\left[\Phi^{\star} \wedge \Phi^{\star}\right]=: \kappa^{\star} .
$$

This two-form can be recognised from (2.53) as the curvature of the connection $\Phi^{\star}$. According to (2.56), it depends on space through $\omega_{\Lambda}$ or, equivalently, through $I$ only. This property stems from the constraints of area and vorticity preservation imposed on the particle motion along vorticity contours. The average in (4.25) is therefore superfluous, and we obtain the result:

P2. The geometric angle of a particle caused by the slow deformation of the boundary is given by

$$
\Delta \theta_{\text {geo }}=\frac{\mathrm{d}}{\mathrm{d} I} \int_{\mathcal{S}_{\Lambda}} \kappa^{\star},
$$

where $\kappa^{\star}$, given in (4.26), is the curvature of $\Phi^{\star}$ and depends only on the action $I$ of the particle and on the domain shape parameterized by $\Lambda$.

\section{Small boundary deformation}

As a concrete illustration of the developments so far, we consider the case where the total boundary deformation is small. In general, computing $g_{\Lambda}$ in (2.24) requires either solving (2.29) or integrating the differential equation (2.18) with boundary condition $\phi=b$ [cf. (2.44)], which would have to be done numerically. Analytic progress is possible, however, if one considers boundary 
deformations that are sufficiently small for a perturbative approach to be applicable. In this section we develop such an approach systematically using Lie series (e.g., [13]).

Let $\delta$ be a formal small parameter characterising the smallness of the boundary deformation. The function $B(\mathbf{x} ; \Lambda)$ defining $D_{\Lambda}$ can then be expanded as

$$
B(\mathbf{x} ; \Lambda)=B_{0}(\mathbf{x})+\delta B_{1}(\mathbf{x} ; \Lambda)+\delta^{2} B_{2}(\mathbf{x} ; \Lambda)+\cdots,
$$

where $B_{0}$ is independent of $\Lambda$ (recall that $\Lambda_{0}=0$ ), $B_{1}$ is linear in $\Lambda, B_{2}$ quadratic, etc. Since $g_{\Lambda}$ is close to the identity and area preserving, it may be regarded as the flow at 'time' $\delta$ of an associated $\delta$-dependent divergence-free vector field given by $\nabla^{\perp} \rho$ for some function $\rho(\mathbf{x}, \delta)$, with $\rho \in \mathcal{C}\left(\mathbb{R}^{2} \times \mathbb{R}\right)$. Correspondingly, the pull-back of $g_{\Lambda}$ defined in (2.26) satisfies

$$
\frac{\mathrm{d} g_{\Lambda}^{*}}{\mathrm{~d} \delta}=g_{\Lambda}^{*}[\rho, \cdot]
$$

Expanding $\rho$ in powers of $\delta$ as

$$
\rho=\rho_{1}+\delta \rho_{2}+\cdots
$$

and introducing this into (5.2) lead to the expansions

$$
\begin{aligned}
& g_{\Lambda}^{*}=1+\delta\left[\rho_{1}, \cdot\right]+\frac{\delta^{2}}{2}\left(\left[\rho_{2}, \cdot\right]+\left[\rho_{1},\left[\rho_{1}, \cdot\right]\right]\right)+\cdots \\
& \left(g_{\Lambda}^{-1}\right)^{*}=1-\delta\left[\rho_{1}, \cdot\right]-\frac{\delta^{2}}{2}\left(\left[\rho_{2}, \cdot\right]-\left[\rho_{1},\left[\rho_{1}, \cdot\right]\right]\right)+\cdots
\end{aligned}
$$

Computing $g_{\Lambda}^{*} f$ for an arbitrary $\Lambda$-independent $f$ using (5.4), taking the exterior derivative and noting that

$$
\mathbf{d} g_{\Lambda}^{*} f=\left[\Phi, g_{\Lambda}^{*} f\right]
$$

lead to

$$
\Phi=\delta \mathbf{d} \rho_{1}+\frac{\delta^{2}}{2}\left(\mathbf{d} \rho_{2}+\left[\mathbf{d} \rho_{1}, \rho_{1}\right]\right)+\cdots,
$$

up to an arbitrary function of $\omega_{\Lambda}$, after using the Jacobi identity.

Introducing (5.4), (5.5) into (2.29) leads to a sequence of partial differential equations for the coefficients of $\rho$. The first two read

$$
\begin{aligned}
& \left(\Delta-F_{0}^{\prime}\right)\left[\rho_{1}, \psi_{0}\right]-\Delta \chi_{1}=0, \\
& \left(\Delta-F_{0}^{\prime}\right)\left[\rho_{2}, \psi_{0}\right]-2 \Delta \chi_{2}=-2\left[\rho_{1}, \Delta\left[\rho_{1}, \psi_{0}\right]\right]+\left[\rho_{1},\left[\rho_{1}, \omega_{0}\right]\right]+\Delta\left[\rho_{1},\left[\rho_{1}, \psi_{0}\right]\right]+2\left[\rho_{1}, \Delta \chi_{1}\right]-2 \Delta\left[\rho_{1}, \chi_{1}\right],
\end{aligned}
$$

where $F_{0}^{\prime}$ is shorthand for $F_{0}^{\prime} \circ \psi_{0}$, and we have introduced the expansion

$$
G_{\Lambda} \circ \omega_{0}=\psi_{0}+\delta \chi_{1}+\delta^{2} \chi_{2}, \cdots
$$

with $\chi_{n}, n=1,2 \cdots$, depending on $\mathbf{x}$ through $\omega_{0}(\mathbf{x})$. These equations are supplemented by the boundary conditions

$$
\begin{aligned}
& {\left[\rho_{1}, B_{0}\right]=-B_{1},} \\
& {\left[\rho_{2}, B_{0}\right]=-2 B_{2}-2\left[\rho_{1}, B_{1}\right]-\left[\rho_{1},\left[\rho_{1}, B_{0}\right]\right],}
\end{aligned}
$$

to be applied on the curve $B_{0}(\mathbf{x})=B(\mathbf{x} ; 0)=0$. The formulation is then relatively simple, with all the equations to be solved in the original domain $D_{0}$. The functions $\chi_{n}, n=1,2, \ldots$ are found from solvability conditions. These can be made explicit using the same method as in the treatment of (2.43). For instance, using the projection operator $\mathrm{P}_{0}$ associated with lines of constant $\psi_{0}$, (5.8) can be rewritten as

$$
\left(\Delta-F_{0}^{\prime} \mathrm{P}_{0}\right) \varphi=0
$$

where $\varphi:=\left[\rho_{1}, \psi_{0}\right]-\chi_{1}$, implying that

$$
\left[\rho_{1}, \psi_{0}\right]=\mathrm{P}_{0} \varphi \quad \text { and } \quad \chi_{1}=\left(1-\mathrm{P}_{0}\right) \varphi .
$$

Once the $\rho_{n}, n=1,2, \ldots$, are computed, the leading-order vorticity and streamfunction follow readily from

$$
\begin{aligned}
& \omega_{\Lambda}=\omega_{0}-\delta\left[\rho_{1}, \omega_{0}\right]-\frac{\delta^{2}}{2}\left(\left[\rho_{2}, \omega_{0}\right]-\left[\rho_{1},\left[\rho_{1}, \omega_{0}\right]\right]\right)+\cdots, \\
& \psi_{\Lambda}=\psi_{0}-\delta\left(\left[\rho_{1}, \psi_{0}\right]-\chi_{1}\right)-\frac{\delta^{2}}{2}\left(\left[\rho_{2}, \psi_{0}\right]-\left[\rho_{1},\left[\rho_{1}, \psi_{0}\right]\right]-2 \chi_{2}\right)+\cdots .
\end{aligned}
$$


To find the first-order correction to the Eulerian flow, (3.8) needs to be solved by expansion in powers of $\delta$. This is conveniently done by pulling back this equation to the original domain $D_{0}$. To do this, we define the pull-backs (denoted by overbars) and their expansions as

$$
\begin{aligned}
& \bar{\Psi}^{(1)}:=g_{\Lambda}^{*} \Psi^{(1)}=\delta \bar{\Psi}_{1}^{(1)}+\delta^{2} \bar{\Psi}_{2}^{(1)}+\cdots, \\
& \bar{\Phi}:=g_{\Lambda}^{*} \Phi=\delta \bar{\Phi}_{1}+\delta^{2} \bar{\Phi}_{2}+\cdots \\
& =\delta \mathbf{d} \rho_{1}+\frac{\delta^{2}}{2}\left(\mathbf{d} \rho_{2}-\left[\mathbf{d} \rho_{1}, \rho_{1}\right]\right)+\cdots,
\end{aligned}
$$

where the last equality follows from (5.4) and (5.7). Introducing these pull-backs into (3.8) and noting that

$$
\begin{aligned}
\left(g_{\Lambda}^{-1}\right)^{*}\left(F_{\Lambda}^{\prime} \circ \psi_{\Lambda}\right) & =F_{\Lambda}^{\prime} \circ \psi_{0}=1 /\left(G_{\Lambda}^{\prime} \circ \omega_{0}\right) \\
& =F_{0}^{\prime} \circ \psi_{0}-\delta\left(F_{0}^{\prime} \circ \psi_{0}\right)^{2} \frac{\nabla \chi_{1}}{\nabla \omega_{0}}+\cdots
\end{aligned}
$$

lead to

$$
\begin{aligned}
& \left(\Delta-F_{0}^{\prime} \mathrm{P}_{0}\right) \bar{\Psi}_{1}^{(1)}=-F_{0}^{\prime} \mathrm{P}_{0} \bar{\Phi}_{1}, \\
& \left(\Delta-F_{0}^{\prime} \mathrm{P}_{0}\right) \bar{\Psi}_{2}^{(1)}=-F_{0}^{\prime} \mathrm{P}_{0} \bar{\Phi}_{2}+\Delta\left[\rho_{1}, \bar{\Psi}_{1}^{(1)}\right]-\left[\rho_{1}, \Delta \bar{\Psi}_{1}^{(1)}\right]-F_{0}^{\prime} \frac{\nabla \chi_{1}}{\nabla \omega_{0}} \Delta \bar{\Psi}_{1}^{(1)} .
\end{aligned}
$$

These equations, involving the same invertible operator as (5.13), can be solved to find $\bar{\Psi}^{(1)}$, with $\Psi^{(1)}$ deduced after application of $\left(g_{\Lambda}^{-1}\right)^{*}$. The natural gauge $\Phi^{\star}$ of $\Phi$ then follows from (3.5) and (3.10). Alternatively, one can first compute the pull-back $\bar{\Phi}^{\star}$, which is obtained from the relations

$$
\bar{\Phi}^{\star}=\mathrm{P}_{0} \bar{\Phi}+\Pi^{\star} \circ \omega_{0} \quad \text { and } \quad \Pi^{\star} \circ \omega_{0}=\left(1-\mathrm{P}_{0}\right) \bar{\Psi}^{(1)}
$$

inferred from (3.5) and (3.10), and then deduce $\Phi^{\star}$ by pushing forward with $\left(g_{\Lambda}^{-1}\right)^{*}$.

To compute the curvature $\kappa^{\star}$ and the geometric angle, there is in fact no need to push forward $\bar{\Psi}^{(1)}$ and $\bar{\Phi}^{\star}$ : indeed, from (4.7) and (4.21), we see that $\hat{\Psi}^{(1)}$ and $\bar{\Psi}^{(1)}$ are related by the $\Lambda$-independent transformation

$$
\hat{\Psi}^{(1)}(I, \theta ; \Lambda)=\bar{\Psi}^{(1)}(\mathbf{X}(I, \theta ; 0) ; \Lambda)
$$

defining the action-angle variables in the original domain $D_{0}$. Since $\hat{\Phi}^{\star}$ and $\bar{\Phi}^{\star}$ obey an analogous relation, they are essentially equivalent: in particular, $\mathbf{d} \hat{\Phi}^{\star}=\mathbf{d} \bar{\Phi}^{\star}$ and $\left[\hat{\Phi}^{\star} \wedge \hat{\Phi}^{\star}\right]=\left[\bar{\Phi}^{\star} \wedge \bar{\Phi}^{\star}\right]$. The curvature $\kappa^{\star}$ in (4.26) can therefore be computed directly from $\bar{\Phi}^{\star}$ in a straightforward manner as

$$
\kappa^{\star}=\mathbf{d} \bar{\Phi}^{\star}-\frac{1}{2}\left[\bar{\Phi}^{\star} \wedge \bar{\Phi}^{\star}\right] .
$$

Note that, in principle, the first two terms in the expansion of $\bar{\Phi}^{\star}$ or $\Phi^{\star}$ need to be computed in order to obtain a leading-order approximation to the geometric angle. This is because $\bar{\Phi}_{1}^{\star}$ is independent of $\Lambda, \mathbf{d} \bar{\Phi}_{1}^{\star}=0$ and hence $\kappa^{\star}=O\left(\delta^{2}\right)$. The computation can however be shortened by observing that the average of $\bar{\Phi}_{2}^{\star}$ along streamlines, that is, $\left(1-\mathrm{P}_{0}\right) \bar{\Phi}_{2}^{\star}$, is the only $O\left(\delta^{2}\right)$ quantity genuinely needed if the averaged form $(4.25)$ of $\kappa^{\star}$ is used. In turn, $\left(1-\mathrm{P}_{0}\right) \bar{\Phi}_{2}^{\star}$ can be approximated by $\left(1-\mathrm{P}_{0}\right) \bar{\Psi}_{2}^{(1)}$, as the pull-back of (3.8) indicates. The latter quantity satisfies a relatively simple equation,

$$
\left(1-\mathrm{P}_{0}\right) \Delta \bar{\Psi}_{2}^{(1)}=\left(1-\mathrm{P}_{0}\right)\left\{\Delta\left[\rho_{1}, \bar{\Psi}_{1}^{(1)}\right]-\left[\rho_{1}, \Delta \bar{\Psi}_{1}^{(1)}\right]\right\},
$$

which is obtained by applying $\left(1-\mathrm{P}_{0}\right)$ to $(5.20)$ and using $\left(1-\mathrm{P}_{0}\right) \Delta \bar{\Psi}_{1}^{(1)}=0$, with the latter relation resulting from (3.3) at leading order in $\delta$.

\section{Nearly axisymmetric flow}

We now consider a simple example where the computations of $g_{\Lambda}$ and other relevant quantities can be carried out explicitly to $O\left(\delta^{2}\right)$. We assume that for $\Lambda=0$, the fluid domain is the disc $(r, \sigma) \in[0,1] \times[0,2 \pi]$. The deformed domain is defined in the form (2.1) by

$$
r=1+\delta \sum_{m} \Lambda_{m} \mathrm{e}^{\mathrm{i} m \sigma}-\frac{\delta^{2}}{2} \sum_{m}\left|\Lambda_{m}\right|^{2}+O\left(\delta^{3}\right),
$$


where the $\Lambda_{m} \in \mathbb{C}$ satisfy $\Lambda_{m}^{*}=\Lambda_{-m}$. Area preservation at $O\left(\delta^{2}\right)$ requires that $\Lambda_{0}=0$ and the introduction of the $O\left(\delta^{2}\right)$, $\sigma$-independent terms.

\subsection{Arbitrary axisymmetric flow}

The initial flow is taken to be axisymmetric, with vorticity

$$
\omega_{0}(r)=\frac{1}{r}\left(r \psi_{0}^{\prime}(r)\right)^{\prime},
$$

where the prime denotes differentiation with respect to $r$. For this flow, (5.8) reduces to

$$
\frac{\psi_{0}^{\prime}}{r} \Delta \partial_{\sigma} \rho_{1}+2\left(\frac{\psi_{0}^{\prime}}{r}\right)^{\prime}\left(\partial_{r \sigma}^{2} \rho_{1}-\frac{1}{r} \partial_{\sigma} \rho_{1}\right)+\frac{1}{r}\left(r \chi_{1}^{\prime}\right)^{\prime}=0
$$

with $\chi_{1}$ a function of $r$ only. The corresponding boundary condition is obtained from (5.11) in the form

$$
\partial_{\sigma} \rho_{1}=-\sum_{m} \Lambda_{m} \mathrm{e}^{\mathrm{i} m \sigma} \quad \text { at } r=1
$$

The solvability condition for (6.2), found by integration with respect to $\sigma \in[0,2 \pi]$, imposes that $\left(r \chi_{1}^{\prime}\right)^{\prime}=0$; boundedness of $\chi_{1}$ then implies that $\chi_{1}$ is a constant which we can take equal to zero: $\chi_{1}=0$.

The solution of (6.2) for $\rho_{1}$ is then found as the Fourier series

$$
\rho_{1}(r, \sigma)=\sum_{m} \Lambda_{m} \rho_{1, m}(r) \mathrm{e}^{\mathrm{i} m \sigma}
$$

with $\rho_{1, m}^{*}=\rho_{1,-m}$. Equation (6.2) does not constrain the $m=0$ mode $\rho_{1,0}$; this is the result of the gauge freedom for $g_{\Lambda}$. A convenient choice is

$$
\rho_{1,0}=0 .
$$

Introducing (6.4) into (6.2) gives a second-order equation for $\rho_{1, m}$, namely

$$
\psi_{0}^{\prime}\left(\rho_{1, m}^{\prime \prime}-\frac{1}{r} \rho_{1, m}^{\prime}+\frac{2-m^{2}}{r^{2}} \rho_{1, m}\right)+2 \psi_{0}^{\prime \prime}\left(\rho_{1, m}^{\prime}-\frac{1}{r} \rho_{1, m}\right)=0,
$$

with associated boundary condition

$$
\rho_{1, m}=\frac{\mathrm{i}}{m} \quad \text { at } r=1 .
$$

There is a close connection between this equation and the Rayleigh equation for the normal modes of axisymmetric flows (e.g., [6]): (6.6) can be recast as the Rayleigh equation for zero-frequency modes, with $r^{-1} \psi_{0}^{\prime} \rho_{1, m}$ as the unknown function. Of course the nonhomogeneous boundary condition for $\rho_{1, m}$ differs from the homogeneous boundary condition usually considered for the Rayleigh equation. The connection is useful nevertheless: the absence of zero-frequency normal modes that can be established from the Rayleigh equation when $\psi_{0}^{\prime} \neq 0$ (as guaranteed by the Hypothesis H2) implies the existence of a unique solution to (6.6).

We note that the solution for the $m=1$ mode, which describes a rigid translation of the disc, is independent of $\psi_{0}$ and given by $\rho_{1, \pm 1}=\mathrm{i} \Lambda_{ \pm 1} r$. Not surprisingly, this corresponds to a uniform displacement field $\nabla^{\perp} \rho_{1}$.

The vanishing of $\chi_{1}$ indicates that the vorticity-stream function relationship is unchanged at leading order in $\delta$. This is a particularity of axisymmetric flows which makes it worthwhile to carry out the calculation to $O\left(\delta^{2}\right)$ so as to demonstrate how a non-zero $\chi_{2}$ is obtained; this is described in Appendix D.

With $\rho_{1}$ determined by its Fourier series $(6.4), \bar{\Phi}_{1}$ is given by

$$
\bar{\Phi}_{1}=\sum_{m} \rho_{1, m}(r) \mathrm{e}^{\mathrm{i} m \sigma} \mathbf{d} \Lambda_{m}
$$

Since $\rho_{1,0}=0$, and $1-\mathrm{P}_{0}$ is simply the average along circles, (5.19) indicates that $\left(1-\mathrm{P}_{0}\right) \bar{\Psi}_{1}^{(1)}=0$. Equations (5.21) then imply that $\bar{\Phi}_{1}^{\star}=\bar{\Phi}_{1}$. In other words, our choice (6.5) provides the leading-order connection with its natural choice of gauge which corresponds to vanishing average along the circles $r=$ const. Expanding $\bar{\Psi}_{1}^{(1)}$ in Fourier series as

$$
\bar{\Psi}_{1}^{(1)}=\sum_{m} \bar{\Psi}_{1, m}^{(1)}(r) \mathrm{e}^{\mathrm{i} m \sigma} \mathbf{d} \Lambda_{m}
$$


(5.19) is reduced to the set of ordinary differential equations

$$
\psi_{0}^{\prime}\left[\frac{1}{r}\left(r \bar{\Psi}_{1, m}^{(1)}\right)^{\prime}-\frac{m^{2}}{r} \bar{\Psi}_{1, m}^{(1)}\right]-\omega_{0}^{\prime} \bar{\Psi}_{1, m}^{(1)}=-\omega_{0}^{\prime} \rho_{1, m}
$$

with $\bar{\Psi}_{1,0}^{(1)}=0$. The associated boundary conditions are found from (3.9) as

$$
\bar{\Psi}_{1, m}^{(1)}=\frac{\mathrm{i}}{m} \quad \text { at } r=1 .
$$

Solving (6.10) gives the first-order correction $\Psi_{1}^{(1)}$ to the Eulerian flow to leading order in $\delta$.

As discussed at the end of Section 5, the computation of the geometric angle to leading order requires not only $\bar{\Phi}_{1}^{\star}$ but also $\bar{\Phi}_{2}^{\star}$ or, to minimise computations, $\left(1-\mathrm{P}_{0}\right) \bar{\Psi}_{2}^{(1)}$. This is deduced from (5.24) which reduces to the ordinary differential equation

$$
\frac{1}{r} \frac{\mathrm{d}}{\mathrm{d} r}\left\{r \frac{\mathrm{d}}{\mathrm{d} r}\left(1-\mathrm{P}_{0}\right) \bar{\Psi}_{2}^{(1)}\right\}=\left(1-\mathrm{P}_{0}\right)\left\{\Delta\left[\rho_{1}, \bar{\Psi}_{1}^{(1)}\right]-\left[\rho_{1}, \Delta \bar{\Psi}_{1}^{(1)}\right]\right\} .
$$

Solving this equation leads to an expression for $\left(1-\mathrm{P}_{0}\right) \bar{\Psi}_{2}^{(1)}$. Taking the differential yields the first term of the curvature $\kappa^{\star}$ in (5.23) as

$$
\left\langle\mathbf{d} \bar{\Phi}^{\star}\right\rangle=\delta^{2}\left(1-\mathrm{P}_{0}\right) \mathbf{d} \bar{\Psi}_{2}^{(1)}+O\left(\delta^{3}\right) .
$$

Note that since $\rho_{1}$ is linear in $\Lambda_{m}$ and $\bar{\Psi}_{1}^{(1)}$ is $\Lambda$-independent, $\left(1-\mathrm{P}_{0}\right) \bar{\Psi}_{2}^{(1)}$ is linear in $\Lambda_{m}$; furthermore, because the averaging $1-\mathrm{P}_{0}$ along circles eliminates all products in the right-hand side of (6.12) except for those of complex-conjugate Fourier modes, $\left(1-\mathrm{P}_{0}\right) \bar{\Psi}_{2}^{(1)}$ is a linear combination of terms of the type $\Lambda_{m} \mathbf{d} \Lambda_{m}^{*}$. Therefore, $\left\langle\mathbf{d} \bar{\Phi}^{\star}\right\rangle$ is given by a $\Lambda$-independent linear combination of the two-forms $\mathbf{d} \Lambda_{m} \wedge \mathbf{d} \Lambda_{m}^{*}$.

The second term in (5.23) is also $O\left(\delta^{2}\right)$ and is readily computed from (6.8). Averaging along circles gives it the same form as that of $\left\langle\mathbf{d} \bar{\Phi}^{\star}\right\rangle$. This leads to the geometric angle in the form

$$
\Delta \theta_{\text {geo }}=\delta^{2} \sum_{m>0} f_{m}(r) \mathcal{A}_{m}+O\left(\delta^{3}\right)
$$

for some functions $f_{m}(r)$. Here we have defined

$$
\mathcal{A}_{m}=-\frac{\mathrm{i}}{2} \int_{\mathcal{D}_{\Lambda}} \mathbf{d} \Lambda_{m} \wedge \mathbf{d} \Lambda_{m}^{*}
$$

which be recognised as (minus) the oriented area enclosed by the path described by $\Lambda_{m}$ in the complex plane. (A positive $\mathcal{A}_{m}$ is associated with a rotation of the fluid domain in the positive sense.) Unsurprisingly, at leading order, the geometric angle is the sum of separate contributions of each Fourier mode of the boundary deformation.

\subsection{An example: Flow with power-law radial dependence}

As a simple example of an axisymmetric flow, consider the streamfunction

$$
\psi_{0}(r)=A r^{\alpha} \quad \text { with } 0<\alpha<2,
$$

for which (6.6)-(6.7) can be solved explicitly, leading to

$$
\bar{\Phi}_{1, m}=\rho_{1, m}=\frac{\mathrm{ir}^{\beta_{m}}}{m}
$$

where

$$
\beta_{m}=\alpha_{m}-\alpha+2 \text { and } \alpha_{m}=\sqrt{m^{2}+\alpha^{2}-2 \alpha} .
$$

The leading-order vorticity in the deformed domain is then found to be

$$
\omega_{\Lambda}(r, \sigma)=\omega_{0}(r)-\delta \omega_{0}^{\prime}(r) \sum_{m} \Lambda_{m} r^{\beta_{m}-1} \mathrm{e}^{\mathrm{i} m \sigma}+O\left(\delta^{2}\right)
$$

with a similar expression for $\psi_{\Lambda}$. Fig. 3 shows these approximations to $\omega_{\Lambda}$ and $\psi_{\Lambda}$ in domains deformed by a single Fourier mode $m$, with $m$ ranging from 2 to 6 , in the case $\alpha=1 / 2$. 


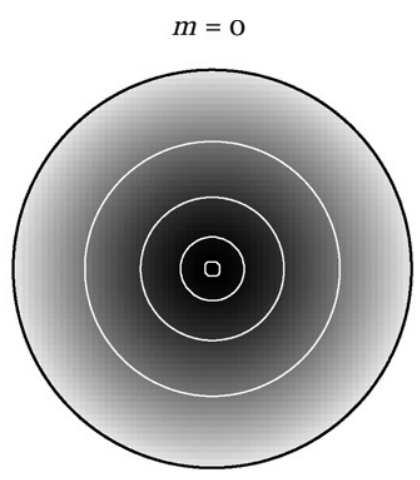

$m=3$

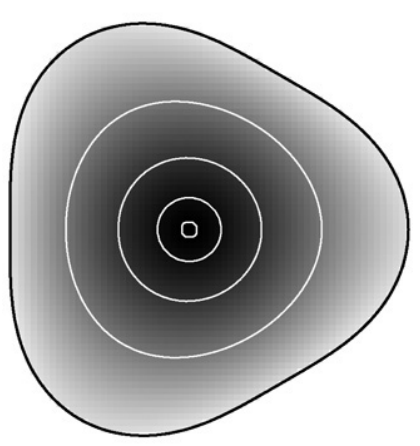

$m=5$

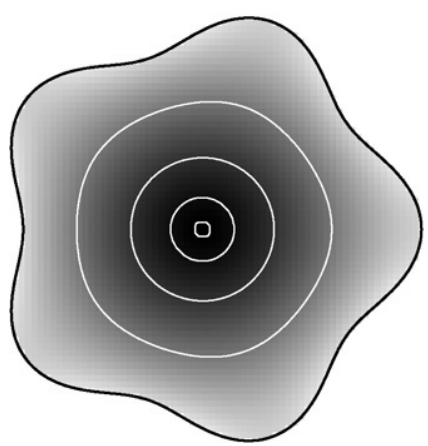

$m=2$

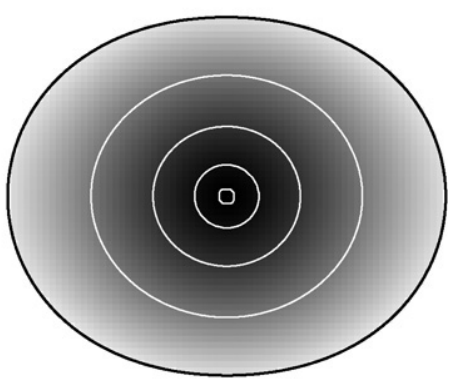

$m=4$

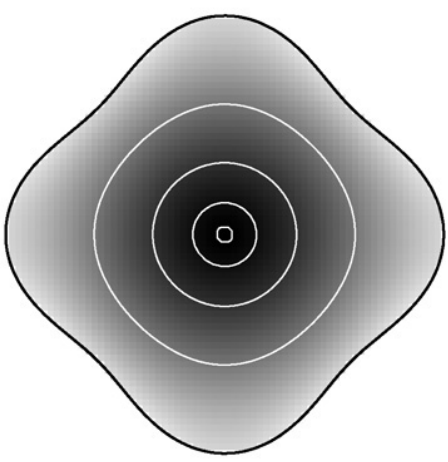

$m=6$

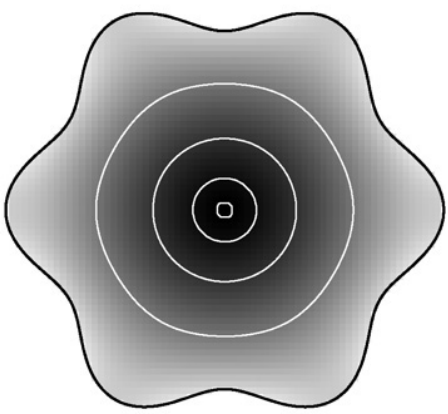

Fig. 3. Vorticity $\omega_{\Lambda}$ (grey scale) and streamfunction $\psi_{\Lambda}$ (white lines) of the leading-order (steady) flow in a slightly deformed disc. The top left panel $m=0$ shows the undeformed flow, with $\psi_{0}=r^{1 / 2}$; the other panels show the flows obtained when deforming the disc by a single Fourier mode $m$ according to (6.1) with $\delta\left|\Lambda_{m}\right|=0.05$.

Eq. (6.10) can also be solved explicitly, with the result

$$
\bar{\Psi}_{1, m}^{(1)}=\frac{\mathrm{i}}{m}\left[\gamma_{m} r^{\beta_{m}}+\left(1-\gamma_{m}\right) r^{\alpha_{m}}\right], \quad \text { where } \gamma_{m}=\frac{\alpha}{\alpha_{m}+\beta_{m}}
$$

Introducing into (6.12) gives

$$
\left(1-\mathrm{P}_{0}\right) \bar{\Psi}_{2}^{(1)}=-\mathrm{i} \sum_{m} \frac{1}{m} \Lambda_{m} \mathbf{d} \Lambda_{m}^{*}\left[\gamma_{m} F\left(\beta_{m}, \beta_{m}\right) r^{2 \beta_{m}-2}+\left(1-\gamma_{m}\right) F\left(\alpha_{m}, \beta_{m}\right) r^{\alpha_{m}+\beta_{m}-2}\right],
$$

where we have defined

$$
F\left(\alpha_{m}, \beta_{m}\right):=\frac{E\left(\alpha_{m}, \beta_{m}\right)}{\alpha_{m}+\beta_{m}-2}:=\frac{2 \alpha_{m} \beta_{m}+\beta_{m}^{2}-2 \alpha_{m}-2 \beta_{m}+m^{2}}{\alpha_{m}+\beta_{m}-2} .
$$

From this and (6.13) we deduce the first component of $\kappa^{\star}$, namely

$$
\left\langle\mathbf{d} \bar{\Phi}^{\star}\right\rangle=-2 \mathrm{i} \delta^{2} \sum_{m>0} \frac{1}{m}\left[\gamma_{m} F\left(\beta_{m}, \beta_{m}\right) r^{2 \beta_{m}-2}+\left(1-\gamma_{m}\right) F\left(\alpha_{m}, \beta_{m}\right) r^{\alpha_{m}+\beta_{m}-2}\right] \mathbf{d} \Lambda_{m} \wedge \mathbf{d} \Lambda_{m}^{*}+O\left(\delta^{3}\right) .
$$




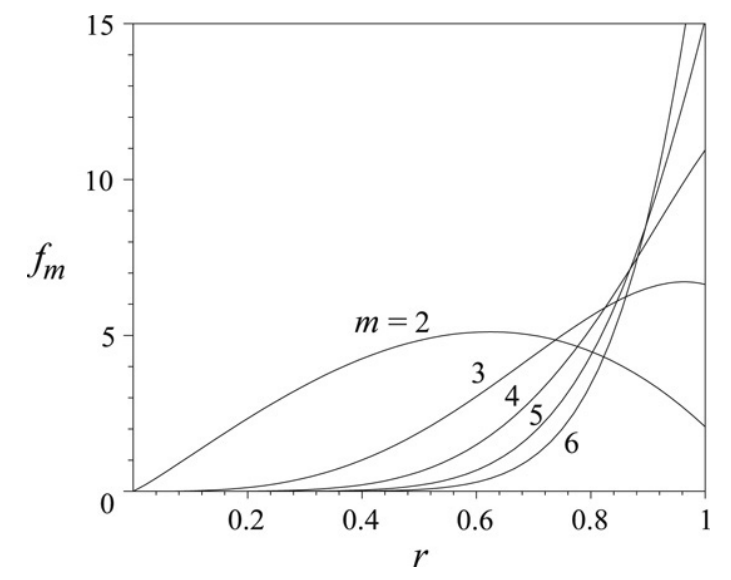

Fig. 4. Functions $f_{m}(r)$ giving the geometric angle in (6.14) for the axisymmetric flow with streamfunction $\psi_{0}=r^{1 / 2}$ in a disc deformed by a Fourier mode $m$.

Using (6.16), the second component of $\kappa^{\star}$ is found directly to be

$$
\begin{aligned}
\left\langle\left[\bar{\Phi}^{\star} \wedge \bar{\Phi}^{\star}\right]\right\rangle & =\frac{\delta^{2}}{2 \pi} \int_{0}^{2 \pi}\left[\bar{\Phi}_{1} \wedge \bar{\Phi}_{1}\right] \mathrm{d} \sigma+O\left(\delta^{3}\right) \\
& =-4 \mathrm{i} \delta^{2} \sum_{m>0} \frac{\beta_{m}}{m} r^{2 \beta_{m}-2} \mathbf{d} \Lambda_{m} \wedge \mathbf{d} \Lambda_{m}^{*}+O\left(\delta^{3}\right) .
\end{aligned}
$$

Combining these results with (4.25), and noting that the action-angle variables in the undeformed domain are simply $(I, \theta)=$ $\left(r^{2} / 2, \sigma\right)$, lead to the geometric angle in the form (6.14) with

$$
f_{m}(r)=\frac{4}{m}\left(p_{m} r^{2 \beta_{m}-4}+q_{m} r^{\alpha_{m}+\beta_{m}-4}\right),
$$

where

$$
p_{m}=\gamma_{m} E\left(\beta_{m}, \beta_{m}\right)-2 \beta_{m}\left(\beta_{m}-1\right) \quad \text { and } \quad q_{m}=\left(1-\gamma_{m}\right) E\left(\alpha_{m}, \beta_{m}\right) .
$$

Fig. 4 shows the functions $f_{m}(r)$ for $m=2,3, \ldots, 6$ in the case $\alpha=1 / 2$.

A spot check for our results is provided by the limit $\alpha \rightarrow 2$, corresponding to a flow with a uniform vorticity $4 A$. Assuming that $\Lambda_{m}=0$ if $m \neq \pm 2$ and that $\Lambda_{ \pm 2}$ traces the unit circle in the complex plane, the domain deformation is simply the rotation of a small-eccentricity ellipse with semi-axes $1+2 \delta$ and $1-2 \delta$. There is an exact analytic solution for such a uniform-vorticity flow in a rotating ellipse; both the direct use of this solution and (6.14) yield the same $r$-independent value for the leading-order geometric angle that appears for one full rotation of the ellipse, namely $16 \pi \delta^{2}$. See Appendix E for details.

\section{Discussion}

In this paper, we have developed a framework to study the dynamics of $2 \mathrm{~d}$ perfect fluids as the boundary of their domain is deformed slowly. The main results are given in Propositions P1 and P2 which characterise the leading-order evolution of the Eulerian and Lagrangian flows. The framework makes explicit use of the fact that the leading-order vorticity is a rearrangement of the initial vorticity. This is done by introducing the diffeomorphism $g_{\Lambda}$ which affects this rearrangement. The differential of $g_{\Lambda}$ with respect to the parameters which define the shape of the boundary, identified as the connection form $\Phi$, is then shown to play a central role in the Lagrangian problem. Although our formulation is in principle valid for arbitrary domain deformations, as long as the hypotheses $\mathrm{H} 1-\mathrm{H} 2$ continue to hold, explicit computations are most easily carried out when the deformations are small and when the initial domain (or a reference domain) is a disc. Simplified formulations, taking advantage of these two features in succession, are also developed.

We emphasize that our results are local in nature: the prediction of an instantaneously steady Eulerian flow, for instance, holds only if the domain deformation is such that $\mathrm{H} 1$ and $\mathrm{H} 2$ are always satisfied. This is necessarily the case if they are satisfied initially and the domain deformation is sufficiently small, but it may well continue to hold for larger deformations. It is nonetheless interesting to speculate about the dynamics when either $\mathrm{H} 1$ or $\mathrm{H} 2$ fails in the course of the evolution. If $\mathrm{H} 1$ fails, the flow ceases to be Arnold stable and likely becomes spectrally unstable. We can then expect the flow to become highly unsteady and, in the absence of dissipative mechanisms, remain so regardless of subsequent deformations of the domain.

The failure of $\mathrm{H} 2$, on the other hand, corresponds to the appearance of streamlines for which the orbiting period of particles becomes large. When the period becomes comparable with the timescale of the domain deformation, our asymptotic approach 
clearly breaks down. This can happen when the flow is driven by the domain deformation towards a change in topology, with the creation of hyperbolic stagnation points and separatrices. How the flow evolves in this situation is unclear, but some understanding could be gained by investigating the problem where the initial steady flow $\psi_{0}$ has a hyperbolic stagnation point (and satisfies H1the Kelvin-Stuart vortex in [10] is just one example). This problem can be viewed as a generalisation of the classical critical-layer problem for parallel shear flows (e.g., [24,15] and references therein). In this generalisation, the separatrix plays the role of the zero-velocity critical line (along which $\mathrm{H} 2$ is obviously violated); by analogy, it can be expected to be also surrounded by a narrow critical layer where complicated nonlinear dynamics occurs. We plan to investigate this problem in future work.

A few remarks are in order on the possible extension of our results to flows in three dimensions. In three dimensions, the dynamics of an inviscid and incompressible fluid is determined, as in two dimensions, by a form of conservation of vorticity, although in this case it is as a vector that the vorticity is transported (e.g., [4]). This suggests that our approach for the determination of the leadingorder Eulerian flow in deforming domains can be adapted to the $3 \mathrm{~d}$ setting. The technical conditions for the well-posedness of the equations for $g_{\Lambda}$ are however likely to be significantly more complicated than that in two dimensions.

The evolution of the fluid-particle position seems, at first sight, to pose a very different problem in three than in two dimensions, since the velocity field is divergence-free and not Hamiltonian. However, particle trajectories for (non-Beltrami) steady solutions of the Euler equations are known to be integrable [1,2] because they are confined to surfaces of constant Bernoulli function (Lamb surfaces). There is, therefore, a simple characterisation of the fluid-particle positions in steady flows, analogous to the action-angle characterisation in two dimensions. This could be used for slowly time-dependent flows to quantify the effects of a cyclic boundary deformation as was done to obtain the geometric angle in this paper.

A difficulty with $3 \mathrm{~d}$ flows, however, is the absence of general stability results similar to those obtained by the energy-Casimir method [10]. Instabilities cannot therefore be excluded (on the contrary, they are the rule rather than the exception), and the effect of their competing with the slow evolution of the leading-order flow would need to be assessed carefully.

In this paper, we have used differential geometry mostly as a notational tool, its main application being to make explicit the linear dependence of various quantities on $\dot{\Lambda}$. It is nonetheless clear that the objects we are dealing with can be given an interpretation in a more abstract geometric setting and that the problem may be placed in the framework of geometric mechanics. This would be a valuable undertaking, but one which is probably quite involved due to the infinite-dimensional nature of the problem, and which is certainly beyond the scope of the present paper. In the rest of this section, we discuss briefly and informally the geometric context of our results in order to elucidate the meanings of the connection one-form $\Phi^{\star}$, the form of its curvature $\kappa^{\star}$, etc.

Central to our development is the group $G=\operatorname{SDiff}\left(\mathbb{R}^{2}\right)$ of area-preserving diffeomorphisms on the plane. Taking the initial domain $D_{0}$ as a reference domain, the subgroup $H \subset G$ which maps $D_{0}$ to itself, viz.,

$$
H=\left\{g \in G: g D_{0}=D_{0}\right\},
$$

is of particular importance. In terms of $G$ and $H$, our parameter space $\mathcal{L}$, the space of all possible shapes $\Lambda$ of the domain $D_{\Lambda}$, can be realised as the quotient $G / H$. Indeed, each right coset of $H$ in $G$ contains all diffeomorphisms mapping $D_{0}$ to $D_{\Lambda}$ for a particular $\Lambda$, since any two such diffeomorphisms $g$ and $g^{\prime}$ are related by $g^{\prime}=g h$ for some $h \in H$. We can therefore identify $\mathcal{L}$ with $G / H$. Another important subgroup is

$$
H_{0}=\left\{h \in H: \omega_{0} \circ h=\omega_{0}\right\}
$$

containing all area-preserving diffeomorphisms in $D_{0}$ which leave the initial vorticity distribution invariant. A rearrangement $\omega_{0} \circ g$ of the initial vorticity $\omega_{0}$ can be identified with an element of $G / H_{0}$.

Armed with this setup, we can interpret our results geometrically. The key point is to regard $G$ and $G / H_{0}$ as principal bundles, both with $G / H \simeq \mathcal{L}$ as base manifold. Finding the (leading-order) Eulerian flow for each domain shape $\Lambda$ then amounts to finding a lift from $G / H$ to $G / H_{0}$; finding the Lagrangian particle position amounts to finding a lift from $G / H$ to $G$. $^{1}$ Proposition P1, stating that the leading-order Eulerian flow depends only on the domain shape, says that the lift from $G / H$ to $G / H_{0}$ is path independent; in other words, it defines a section of $G / H_{0}{ }^{2}$ In contrast, the lift from $G / H$ to $G$, which gives an approximation to the particle position, depends on the path in $G / H$ and in fact also on the speed with which the path is traced. There is, however, a contribution that is independent of speed; for cyclic domain deformations, it is quantified by the geometric angle given in P2.

It is worth commenting on the meaning of the one-form $\Phi^{\star}$ that appears in the geometric angle. One way of defining a lift in a principal bundle is by means of a vector-valued one-form, i.e. a linear map from $T(G / H)$ to $T G$, describing the vertical (along-fibre) displacement associated with any given displacement on the base manifold $G / H$. Such a form can be recognised as a connection form. In our context, $T G$ is the space of divergence-free vector fields over $\mathbb{R}^{2}$, which can be identified through the use of a streamfunction with the space of real-valued functions $\mathcal{C}\left(\mathbb{R}^{2}\right)$. Thus, a lift can be defined by a connection one-form over

\footnotetext{
${ }^{1}$ In our geometric description the interior and exterior of $D_{\Lambda}$ are treated on the same footing; this can be done because our formulas, with suitable boundary conditions as $|\mathbf{x}| \rightarrow \infty$, would also apply to a fluid flow outside $D_{\Lambda}$

${ }^{2}$ We stress again the local nature of P1: globally, there are many possible steady flows for a given vorticity distribution and a given domain $D_{\Lambda}$. Geometrically, this implies that the section of $G / H_{0}$ is multivalued.
} 
$G / H \simeq \mathcal{L}$ with values in $\mathcal{C}\left(\mathbb{R}^{2}\right)$. This is precisely the interpretation we give to $\Phi^{\star}$. With the geometric interpretation of $\Phi^{\star}$, the subsequent results are clear: (4.26) is the standard expression for the curvature of $\Phi^{\star}$, the geometric angle (4.27) is given by the holonomy of $\Phi^{\star}$, and the standard conclusion about the geometrical angle in finite-dimensional systems is recovered.

\section{Acknowledgements}

This research was supported by an EPSRC research grant, a William Gordon Seggie Brown Fellowship (DW) and a NERC Advanced Fellowship (JV). Additional support was provided by the EPSRC network "Geometrical method in geophysical fluid dynamics". We thank T.N. Bailey, Y. Brenier and L. Butler for helpful discussions.

\section{Appendix A. Solution of $\left(\Delta-F_{\Lambda}^{\prime} \mathbf{P}_{\Lambda}\right) u=f$}

Here we show that the problem

$$
\begin{aligned}
& \left(\Delta-F_{\Lambda}^{\prime} \mathrm{P}_{\Lambda}\right) \eta=f \\
& \eta=g \quad \text { on } \partial D_{\Lambda}
\end{aligned}
$$

has a unique solution $\eta$ when $F_{\Lambda}^{\prime}>-c_{\text {poi }}$ everywhere in $D_{\Lambda}$ as follows from the Hypothesis H1.

We start with an identity. Let $u$ and $v$ be such that $\mathrm{P}_{\Lambda} u=0$ and $\mathrm{P}_{\Lambda} v=v$. We have

$$
\int_{D_{\Lambda}} u v \mathrm{~d}^{2} \mathbf{x}=\int\{\oint u v \mathrm{~d} s\} \mathrm{d} \psi_{\Lambda}=\int u\{\oint v \mathrm{~d} s\} \mathrm{d} \psi_{\Lambda}=0 .
$$

From this it follows that the projection $\mathrm{P}_{\Lambda}$ is orthogonal in $L^{2}\left(D_{\Lambda}\right)$, in the sense that for any (sufficiently smooth) function $w$,

$$
\begin{aligned}
\int_{D_{\Lambda}}|w|^{2} \mathrm{~d}^{2} \mathbf{x} & =\int_{D_{\Lambda}}\left\{\left|\mathrm{P}_{\Lambda} w\right|^{2}+2\left(\mathrm{P}_{\Lambda} w\right)\left[\left(1-\mathrm{P}_{\Lambda}\right) w\right]+\left|\left(1-\mathrm{P}_{\Lambda}\right) w\right|^{2}\right\} \mathrm{d}^{2} \mathbf{x} \\
& =\int_{D_{\Lambda}}\left\{\left|\mathrm{P}_{\Lambda} w\right|^{2}+\left|\left(1-\mathrm{P}_{\Lambda}\right) w\right|^{2}\right\} \mathrm{d}^{2} \mathbf{x} .
\end{aligned}
$$

Using (A.2), we find that the operator $\left(\Delta-F_{\Lambda}^{\prime} \mathrm{P}_{\Lambda}\right)$ is self-adjoint for any functions $u$ and $v$ which vanish on $\partial D_{\Lambda}$,

$$
\int_{D_{\Lambda}} v\left(\Delta-F_{\Lambda}^{\prime} \mathrm{P}_{\Lambda}\right) u \mathrm{~d}^{2} \mathbf{x}=\int_{D_{\Lambda}} u\left(\Delta-F_{\Lambda}^{\prime} \mathrm{P}_{\Lambda}\right) v \mathrm{~d}^{2} \mathbf{x} .
$$

Moreover, $\left(\Delta-F_{\Lambda}^{\prime} \mathrm{P}_{\Lambda}\right)$ is coercive under the hypothesis $F^{\prime}>-c_{\text {poi }}$. To show this, we first combine (A.3) and Poincaré's inequality to obtain

$$
\int_{D_{\Lambda}}\left|\mathrm{P}_{\Lambda} u\right|^{2} \mathrm{~d}^{2} \mathbf{x} \leq \int_{D_{\Lambda}}|u|^{2} \mathrm{~d}^{2} \mathbf{x} \leq \frac{1}{c_{\text {poi }}} \int_{D_{\Lambda}}|\nabla u|^{2} \mathrm{~d}^{2} \mathbf{x}
$$

for any function $u$ vanishing on $\partial D$. It is then clear that

$$
\int_{D_{\Lambda}} u\left(\Delta-F_{\Lambda}^{\prime} \mathrm{P}_{\Lambda}\right) u \mathrm{~d}^{2} \mathbf{x}=-\int_{D_{\Lambda}}\left\{|\nabla u|^{2}+F_{\Lambda}^{\prime}\left(\mathrm{P}_{\Lambda} u\right)^{2}\right\} \mathrm{d}^{2} \mathbf{x} \leq 0
$$

when $F_{\Lambda}^{\prime}>-c_{\text {poi }}$ everywhere in $D$, obtaining equality only when $u=0$.

Returning to the problem (A.1), we extend $g$ to $\operatorname{cl} D_{\Lambda}$ and let $\tilde{\eta}=\eta-g$. The problem thus becomes

$$
\left(\Delta-F_{\Lambda}^{\prime} \mathrm{P}_{\Lambda}\right) \tilde{\eta}=-\Delta g+F_{\Lambda}^{\prime} \mathrm{P}_{\Lambda} g+f
$$

with boundary conditions $\tilde{\eta}=0$ on $\partial D_{\Lambda}$. We have shown that the operator $\left(\Delta-F_{\Lambda}^{\prime} \mathrm{P}_{\Lambda}\right)$ on the left-hand side is self-adjoint and its associated bilinear form is coercive, so by the Lax-Milgram lemma (assuming compactness, etc., see, e.g., [8]) we can find a unique solution $\tilde{\eta}$ for (A.7) and thus $\eta$ for (A.1).

\section{Appendix B. Properties of $\Phi$}

In this Appendix, we derive some of the properties of the connection form $\Phi$. We begin by establishing the form (2.53) of the curvature of $\Phi$. 


\section{B.1. Curvature form}

The curvature of the connection $\Phi$ encodes the manner in which two diffeomorphisms $g_{\Lambda}$, generated along different paths with the same endpoints, differ. An explicit expression for it is now obtained in a standard fashion, by considering two paths obtained by changing only two components of $\Lambda$, say $\Lambda_{1}$ and $\Lambda_{2}$, by an infinitesimal amount. The two paths chosen join $\left(\Lambda_{1}, \Lambda_{2}\right)=(0,0)$ to $(\delta, \epsilon)$, where $\delta, \epsilon \ll 1$, the first via $(\delta, 0)$ and the second via $(0, \epsilon)$.

For $\tau \in[0,1]$, let us fix a path $\tau \mapsto \gamma(\tau)$ in $\mathcal{L}$ and let $g_{\gamma(\tau)}$ be the diffeomorphism on $\mathbb{R}^{2}$ that is the "flow" of $\Phi$, in the sense that

$$
\frac{\mathrm{d}}{\mathrm{d} t} g_{\gamma(\tau)}=\left(\nabla^{\perp} \Phi \circ g_{\gamma(\tau)}\right) \cdot \dot{\gamma} \quad \text { and } \quad g_{\gamma(0)}=\mathrm{id}
$$

Let $\gamma_{a}(\tau)=(0,0)+(\delta, 0) \tau$ and $g_{a}:=g_{\gamma_{a}(1)}$; similarly, let $\gamma_{b}(\tau)=(\delta, 0)+(0, \epsilon) \tau$ and $g_{b}:=g_{\gamma_{b}(1)}, \gamma_{c}(\tau)=(0,0)+(0, \epsilon) \tau$ and $g_{c}:=g_{\gamma_{c}(1)}, \gamma_{d}(\tau)=(0, \epsilon)+(\delta, 0) \tau$ and $g_{d}:=g_{\gamma_{d}(1)}$. With this notation, the first path is $\gamma_{a} \cup \gamma_{b}$, the second $\gamma_{c} \cup \gamma_{d}$, and in the limit $\delta, \epsilon \rightarrow 0, g_{b} g_{a}-g_{d} g_{c}$ gives ( $\delta \epsilon$ times) one of the components of the curvature of $\Phi$ at $\Lambda=0$.

To compute this component, we first consider the action of $g_{b} g_{a}$ on a function $f$ in $\mathbb{R}^{2}$ and find

$$
f\left(g_{a} \mathbf{x}\right)=f(\mathbf{x})+\delta\left[\Phi_{1}(0,0), f\right](\mathbf{x})+\frac{\delta^{2}}{2}\left[\Phi_{1}(0,0),\left[\Phi_{1}(0,0), f\right]\right](\mathbf{x})+O\left(\epsilon^{3}\right)
$$

and so, with $\hat{f}(\mathbf{x}):=f\left(g_{a} \mathbf{x}\right)$,

$$
\begin{aligned}
f\left(g_{b} g_{a} \mathbf{x}\right)= & \hat{f}(\mathbf{x})+\epsilon\left[\Phi_{2}(\delta, 0), \hat{f}\right](\mathbf{x})+\frac{\epsilon^{2}}{2}\left[\Phi_{2}(\delta, 0),\left[\Phi_{2}(\delta, 0), \hat{f}\right]\right](\mathbf{x})+O\left(\epsilon^{3}\right) \\
= & f(\mathbf{x})+\delta\left[\Phi_{1}, f\right](\mathbf{x})+\frac{\delta^{2}}{2}\left[\Phi_{1},\left[\Phi_{1}, f\right]\right](\mathbf{x})+\epsilon\left[\Phi_{2}, f\right](\mathbf{x})+\epsilon \delta\left[\Phi_{1},\left[\Phi_{2}, f\right]\right](\mathbf{x}) \\
& +\epsilon \delta\left[\frac{\partial \Phi_{2}}{\partial \Lambda_{1}}, f\right](\mathbf{x})+\frac{\epsilon^{2}}{2}\left[\Phi_{2},\left[\Phi_{2}, f\right]\right](\mathbf{x})+\text { h.o.t. },
\end{aligned}
$$

where in the last expression $\Phi_{1}:=\Phi_{1}(0,0), \Phi_{2}:=\Phi_{2}(0,0)$, and h.o.t. denotes cubic and higher terms. Similarly,

$$
\begin{aligned}
f\left(g_{d} g_{c} \mathbf{x}\right)= & f(\mathbf{x})+\epsilon\left[\Phi_{2}, f\right](\mathbf{x})+\frac{\epsilon^{2}}{2}\left[\Phi_{2},\left[\Phi_{2}, f\right]\right](\mathbf{x})+\delta\left[\Phi_{1}, f\right](\mathbf{x})+\delta \epsilon\left[\Phi_{2},\left[\Phi_{1}, f\right]\right](\mathbf{x}) \\
& +\delta \epsilon\left[\frac{\partial \Phi_{1}}{\partial \Lambda_{2}}, f\right](\mathbf{x})+\frac{\delta^{2}}{2}\left[\Phi_{1},\left[\Phi_{1}, f\right]\right](\mathbf{x})+\text { h.o.t. }
\end{aligned}
$$

Subtracting these two expressions, we find

$$
g_{b}^{*} g_{a}^{*} f-g_{d}^{*} g_{c}^{*} f=\delta \epsilon\left\{\left[\Phi_{1},\left[\Phi_{2}, f\right]\right]-\left[\Phi_{2},\left[\Phi_{1}, f\right]\right]+\left[\frac{\partial \Phi_{2}}{\partial \Lambda_{1}}, f\right]-\left[\frac{\partial \Phi_{1}}{\partial \Lambda_{2}}, f\right]\right\}+\text { h.o.t. }
$$

After using the Jacobi identity, $[f,[g, h]]+[g,[h, f]]+[h,[f, g]]=0$ for any three functions $f, g$ and $h$, this can be rearranged as

$$
\left(g_{b} g_{a}-g_{d} g_{c}\right)^{*} f=\delta \epsilon\left[\left\{\frac{\partial \Phi_{2}}{\partial \Lambda_{1}}-\frac{\partial \Phi_{1}}{\partial \Lambda_{2}}+\left[\Phi_{1}, \Phi_{2}\right]\right\}, f\right]+\text { h.o.t. }
$$

From this equation, we can identify the component in $\mathbf{d} \Lambda_{1} \wedge \mathbf{d} \Lambda_{2}$ of the curvature of $\Phi$ as

$$
\frac{\partial \Phi_{2}}{\partial \Lambda_{1}}-\frac{\partial \Phi_{1}}{\partial \Lambda_{2}}+\left[\Phi_{1}, \Phi_{2}\right]
$$

since it describes the diffeomorphism $g_{b} g_{a}-g_{d} g_{c}$ in the limit $\delta, \epsilon \rightarrow 0$. Thus, a general expression for the curvature of $\Phi$ is given by

$$
\kappa=\left\{\frac{\partial \Phi_{n}}{\partial \Lambda_{m}}+\frac{1}{2}\left[\Phi_{m}, \Phi_{n}\right]\right\} \mathbf{d} \Lambda_{m} \wedge \mathbf{d} \Lambda_{n},
$$

which we write in coordinate-free notation as (2.53).

We note that the foregoing development is valid for any connection $\Phi$ and diffeomorphism $g_{\gamma(\tau)}$ satisfying (B.1) since we have made no use of the properties of $\Phi$ and $g_{\Lambda}$ developed elsewhere in this paper. 


\section{B.2. Derivation of (2.55)}

We now derive (2.55). Applying $\mathbf{d}$ to (2.39) gives

$$
\begin{aligned}
0 & =\mathbf{d}^{2} \omega_{\Lambda}=-\mathbf{d}\left[\Phi, \omega_{\Lambda}\right] \\
& =-\left[\mathbf{d} \Phi, \omega_{\Lambda}\right]+\left[\Phi \wedge \mathbf{d} \omega_{\Lambda}\right] \\
& =-\left[\mathbf{d} \Phi, \omega_{\Lambda}\right]-\left[\Phi \wedge\left[\Phi, \omega_{\Lambda}\right]\right] .
\end{aligned}
$$

Now

$$
\begin{aligned}
-\left[\Phi \wedge\left[\Phi, \omega_{\Lambda}\right]\right] & =-\left[\Phi_{m},\left[\Phi_{n}, \omega_{\Lambda}\right]\right] \mathbf{d} \Lambda_{m} \wedge \mathbf{d} \Lambda_{n} \\
& =\left\{\left[\Phi_{n},\left[\omega_{\Lambda}, \Phi_{m}\right]\right]+\left[\omega_{\Lambda},\left[\Phi_{m}, \Phi_{n}\right]\right]\right\} \mathbf{d} \Lambda_{m} \wedge \mathbf{d} \Lambda_{n} \\
& =\left[\Phi \wedge\left[\Phi, \omega_{\Lambda}\right]\right]+\left[\omega_{\Lambda},[\Phi \wedge \Phi]\right]
\end{aligned}
$$

(to arrive at the second equality, we have used the Jacobi identity); therefore

$$
-\left[\Phi \wedge\left[\Phi, \omega_{\Lambda}\right]\right]=\frac{1}{2}\left[\omega_{\Lambda},[\Phi \wedge \Phi]\right]
$$

and from (B.9) we find

$$
\begin{aligned}
& {\left[\mathbf{d} \Phi+\frac{1}{2}[\Phi \wedge \Phi], \omega_{\Lambda}\right]=0 } \\
\Rightarrow & \mathbf{d} \Phi+\frac{1}{2}[\Phi \wedge \Phi]=w \circ \omega_{\Lambda}
\end{aligned}
$$

for an arbitrary function-valued two-form $w \circ \omega_{\Lambda}$. Together with the result of Appendix B.1 above, the first equation implies that the curvature has no effect when considering the actions of diffeomorphisms generated by $\Phi$ on functions of $\omega_{\Lambda}$.

We note that the result (B.12) is valid for any $f_{\Lambda}$ that (i) is a function of $\Lambda$, so $\mathbf{d} f_{\Lambda}$ makes sense, and (ii) satisfies d $f_{\Lambda}+\left[\Phi, f_{\Lambda}\right]=0$.

\section{B.3. Relationship with (B.12)}

Next we show that (B.12) can be derived directly from (2.43). This provides a consistency check for our developments. Let us first compute

$$
\begin{aligned}
\mathbf{d}\left[\Phi, \psi_{\Lambda}\right] & =\mathbf{d}\left\{\left[\Phi_{m}, \psi_{\Lambda}\right] \mathbf{d} \Lambda_{m}\right\} \\
& =\left[\mathbf{d} \Phi_{m}, \psi_{\Lambda}\right] \wedge \mathbf{d} \Lambda_{m}+\left[\Phi_{m}, \mathbf{d} \psi_{\Lambda}\right] \wedge \mathbf{d} \Lambda_{m} \\
& =\left[\mathbf{d} \Phi, \psi_{\Lambda}\right]-\left[\Phi \wedge \mathbf{d} \psi_{\Lambda}\right]
\end{aligned}
$$

and

$$
\begin{aligned}
\mathbf{d}\left(\mathbf{d} G_{\Lambda} \circ \omega_{\Lambda}\right) & =-\left(\mathbf{d} G_{\Lambda}^{\prime} \circ \omega_{\Lambda}\right) \wedge \mathbf{d} \omega_{\Lambda} \\
& =\left(\mathbf{d} G_{\Lambda}^{\prime} \circ \omega_{\Lambda}\right) \wedge\left[\Phi, \omega_{\Lambda}\right] \\
& =\left[\Phi \wedge \mathbf{d} G_{\Lambda}\right],
\end{aligned}
$$

where the last equality can be verified by computation in coordinates.

Writing (2.43) as

$$
\Delta\left[\Phi, \psi_{\Lambda}\right]-\left[\Phi, \omega_{\Lambda}\right]-\Delta\left(\mathbf{d} G_{\Lambda} \circ \omega_{\Lambda}\right)=0
$$

and taking $\mathbf{d}$, we find

$$
\begin{aligned}
0 & =\Delta \mathbf{d}\left[\Phi, \psi_{\Lambda}\right]-\mathbf{d}\left[\Phi, \omega_{\Lambda}\right]-\Delta \mathbf{d}\left(\mathbf{d} G_{\Lambda} \circ \omega_{\Lambda}\right) \\
& =\Delta\left[\mathbf{d} \Phi, \psi_{\Lambda}\right]-\Delta\left[\Phi \wedge \mathbf{d} \psi_{\Lambda}\right]-\left[\mathbf{d} \Phi, \omega_{\Lambda}\right]+\left[\Phi \wedge \mathbf{d} \omega_{\Lambda}\right]+\Delta\left[\Phi \wedge \mathbf{d} G_{\Lambda}\right] \\
& =\left(\Delta-F_{\Lambda}^{\prime}\right)\left[\mathbf{d} \Phi, \psi_{\Lambda}\right]+\Delta\left[\Phi \wedge\left(\mathbf{d} G_{\Lambda}-\mathbf{d} \psi_{\Lambda}\right)\right]+\left[\Phi \wedge \mathbf{d} \omega_{\Lambda}\right] \\
& =\left(\Delta-F_{\Lambda}^{\prime}\right)\left[\mathbf{d} \Phi+\frac{1}{2}[\Phi \wedge \Phi], \psi_{\Lambda}\right] .
\end{aligned}
$$

A couple of identities have been used to arrive at the last equation. The first one is

$$
\left[\Phi \wedge\left[\Phi, \psi_{\Lambda}\right]\right]=\frac{1}{2}\left[[\Phi \wedge \Phi], \psi_{\Lambda}\right]
$$


which is proved in the same way as (B.11). The second identity is

$$
\begin{aligned}
{\left[\Phi \wedge \mathbf{d} \omega_{\Lambda}\right] } & =\left[\Phi \wedge\left[\Phi, F_{\Lambda} \circ \psi_{\Lambda}\right]\right]=\left[\Phi \wedge F_{\Lambda}^{\prime}\left[\Phi, \psi_{\Lambda}\right]\right] \\
& =F_{\Lambda}^{\prime}\left[\Phi \wedge\left[\Phi, \psi_{\Lambda}\right]\right]-\left[\Phi, F_{\Lambda}^{\prime}\right] \wedge\left[\Phi, \psi_{\Lambda}\right]=F_{\Lambda}^{\prime}\left[\Phi \wedge\left[\Phi, \psi_{\Lambda}\right]\right],
\end{aligned}
$$

where we have used $\left[\Phi, F_{\Lambda}^{\prime} \circ \psi_{\Lambda}\right] \wedge\left[\Phi, \psi_{\Lambda}\right]=F_{\Lambda}^{\prime \prime}\left[\Phi, \psi_{\Lambda}\right] \wedge\left[\Phi, \psi_{\Lambda}\right]=0$ for the last equality.

The desired result (B.12) is recovered by noting that the operator $\left(\Delta-F_{\Lambda}^{\prime}\right)$ is invertible by hypothesis and that $\left[\mathbf{d} \Phi+\frac{1}{2}[\Phi \wedge\right.$ $\left.\Phi], \psi_{\Lambda}\right]=0$ on $\partial D_{\Lambda}$. The latter can be verified by differentiating (2.39) and evaluating it on $\partial D_{\Lambda}$.

\section{B.4. Derivation of $(4.26)$}

Finally, we establish the formula

$$
\mathbf{d} \hat{\Phi}-\frac{1}{2}[\hat{\Phi} \wedge \hat{\Phi}]=\mathbf{d} \Phi+\frac{1}{2}[\Phi \wedge \Phi] .
$$

Its application to the natural connection $\Phi^{\star}$ shows that $\kappa^{\star}$ is independent of $\theta$. Our proof starts by noticing that $[\hat{\Phi} \wedge \hat{\Phi}]=[\Phi \wedge \Phi]$ because the transformation to action-angle variables is canonical. Thus (B.19) is equivalent to

$$
\mathbf{d} \hat{\Phi}=\mathbf{d} \Phi+[\Phi \wedge \Phi] .
$$

This is established by direct computation as follows

$$
\begin{aligned}
\mathbf{d} \hat{\Phi}(I, \theta ; \Lambda) & =\mathbf{d} \Phi(\mathbf{X}(I, \theta ; \Lambda) ; \Lambda) \\
& =\mathbf{d} \Phi(\mathbf{x} ; \Lambda)+\left.\left(\mathbf{d X} \cdot \nabla \Phi_{n}\right)\right|_{(\mathbf{x}, \Lambda)} \mathbf{d} \Lambda_{n} \\
& =\mathbf{d} \Phi(\mathbf{x} ; \Lambda)+\left.\left[\Phi_{m}, \Phi_{n}\right]\right|_{(\mathbf{x}, \Lambda)} \mathbf{d} \Lambda_{m} \wedge \mathbf{d} \Lambda_{n} \\
& =\mathbf{d} \Phi(\mathbf{x} ; \Lambda)+\left.[\Phi \wedge \Phi]\right|_{(\mathbf{x}, \Lambda)} .
\end{aligned}
$$

\section{Appendix C. Higher-order streamfunction}

In this Appendix, we compute higher-order corrections to the streamfunction, with the first-order streamfunction $\psi^{(1)}$ derived in Section 3 as a special case. Instead of the expansion (2.8), we use here an iterative procedure. Since much of the computation is similar to earlier developments, we will only sketch the derivation and make references to earlier, analogous steps. We start by writing

$$
\omega=\omega_{\Lambda}+\omega_{\varepsilon} \quad \text { and } \quad \psi=\psi_{\Lambda}+\psi_{\varepsilon}
$$

where $\psi_{\Lambda}=G_{\Lambda}\left(\omega_{\Lambda}\right)$,

$$
\begin{array}{ll}
\omega_{\Lambda}=\Delta \psi_{\Lambda} & \left.\psi_{\Lambda}\right|_{\partial D}=0, \\
\omega_{\varepsilon}=\Delta \psi_{\varepsilon} & \left.\psi_{\varepsilon}\right|_{\partial D}=\varepsilon b,
\end{array}
$$

and $\omega_{\varepsilon}, \psi_{\varepsilon}=O(\varepsilon)$. Since $\omega_{\varepsilon}$ and $\psi_{\varepsilon}$ depend not only on $\dot{\Lambda}$ but also on its derivatives (and nonlinearly), we shall not be using the differential form notation much in this Appendix.

Substituting these into the equation of motion $\varepsilon \partial_{\tau} \omega+[\psi, \omega]=0$, we find after some manipulation [cf. (2.16)-(2.19)]

$$
\varepsilon \partial_{\tau} \omega_{\Lambda}+\left[\phi_{\varepsilon}, \omega_{\Lambda}\right]=-\varepsilon \partial_{\tau} \omega_{\varepsilon}-\left[\psi_{\varepsilon}, \omega_{\varepsilon}\right],
$$

where in analogy with $\phi$ we have defined

$$
\phi_{\varepsilon}:=\left(1-G_{\Lambda}^{\prime} \Delta\right) \psi_{\varepsilon} .
$$

We note that the left-hand side of (C.3) is $O(\varepsilon)$ while the right-hand side is $O\left(\varepsilon^{2}\right)$, assuming that $\omega_{\varepsilon}$ depends slowly on time. Taking $\partial_{\tau}$ of the relation $\psi_{\Lambda}=G_{\Lambda}\left(\omega_{\Lambda}\right)$, we find after some manipulation using (C.3) [cf. (2.42)],

$$
\varepsilon \partial_{\tau} \psi_{\Lambda}+\left[\phi_{\varepsilon}, \psi_{\Lambda}\right]-\chi=-G_{\Lambda}^{\prime} \varepsilon \partial_{\tau} \omega_{\varepsilon}-G_{\Lambda}^{\prime}\left[\psi_{\varepsilon}, \omega_{\varepsilon}\right]
$$

where $\chi:=\left(\mathbf{d} G_{\Lambda} \circ \omega_{\Lambda}\right) \cdot \dot{\Lambda} \in \mathcal{C}\left(D_{\Lambda}\right)$ depends on $\mathbf{x}$ only through $\omega_{\Lambda}$. Taking $\Delta$ of this equation and using (C.3) again, we find after more manipulation [cf. (2.43)]

$$
\left(\Delta-F_{\Lambda}^{\prime}\right)\left[\phi_{\varepsilon}, \psi_{\Lambda}\right]-\Delta \chi=\left(1-\Delta G_{\Lambda}^{\prime}\right)\left\{\varepsilon \partial_{\tau} \omega_{\varepsilon}+\left[\psi_{\varepsilon}, \omega_{\varepsilon}\right]\right\}
$$


Now let $\varphi_{\varepsilon}:=\left[\phi_{\varepsilon}, \psi_{\Lambda}\right]-\chi$. Since $\mathrm{P}_{\Lambda}\left[\phi_{\varepsilon}, \psi_{\Lambda}\right]=\left[\phi_{\varepsilon}, \psi_{\Lambda}\right]$ and $\mathrm{P}_{\Lambda} \chi=0$, we can write (C.6) in the form [cf. (2.50)]

$$
\left(\Delta-F_{\Lambda}^{\prime} \mathrm{P}_{\Lambda}\right) \varphi_{\varepsilon}=\left(1-\Delta G_{\Lambda}^{\prime}\right)\left\{\varepsilon \partial_{\tau} \omega_{\varepsilon}+\left[\psi_{\varepsilon}, \omega_{\varepsilon}\right]\right\},
$$

to which we must add the boundary conditions

$$
\varphi_{\varepsilon}=\left[b, \psi_{\Lambda}\right] \quad \text { on } \partial D_{\Lambda} \text {. }
$$

Once $\varphi_{\varepsilon}$ is known, $\phi_{\varepsilon}$ is determined up to the addition of a function of $\omega_{\Lambda}$ by

$$
\left[\phi_{\varepsilon}, \psi_{\Lambda}\right]=\mathrm{P}_{\Lambda} \varphi_{\varepsilon} \text {. }
$$

To obtain an equation for $\psi_{\varepsilon}$, we need an integral constraint on the vorticity $\omega_{\varepsilon}$. Noting that, for any $f \in \mathcal{C}\left(D_{\Lambda}\right)$,

$$
\int_{D_{\Lambda}} \Theta^{\prime}\left(\omega_{\Lambda}-\Omega\right) f \mathrm{~d}^{2} \mathbf{x}=\oint_{\omega_{\Lambda}=\Omega} f \frac{\mathrm{d} l}{\left|\nabla \omega_{\Lambda}\right|},
$$

and subtracting $\Theta^{\prime}\left(\omega_{\Lambda}-\Omega\right) \omega_{\varepsilon}$ to the integrand on both sides of (3.1), we find

$$
\int_{D_{\Lambda}}\left\{\Theta\left(\omega_{\Lambda}+\omega_{\varepsilon}-\Omega\right)-\Theta^{\prime}\left(\omega_{\Lambda}-\Omega\right) \omega_{\varepsilon}-\Theta\left(\omega_{\Lambda}-\Omega\right)\right\} \mathrm{d}^{2} \mathbf{x}=-\oint_{\omega_{\Lambda}=\Omega} \omega_{\varepsilon} \frac{\mathrm{d} l}{\left|\nabla \omega_{\Lambda}\right|} .
$$

Let us define $\mathcal{E}\left[\omega_{\Lambda}, \omega_{\varepsilon}\right] \in \mathcal{C}\left(D_{\Lambda}\right)$ by

$$
\mathcal{E}\left[\omega_{\Lambda}, \omega_{\varepsilon}\right](\mathbf{x}) \oint_{\omega_{\Lambda}=\Omega} \frac{\mathrm{d} l^{\prime}}{\left|\nabla \omega_{\Lambda}\right|}:=\int_{D_{\Lambda}}\left\{\Theta\left(\omega_{\Lambda}+\omega_{\varepsilon}-\Omega\right)-\Theta^{\prime}\left(\omega_{\Lambda}-\Omega\right) \omega_{\varepsilon}-\Theta\left(\omega_{\Lambda}-\Omega\right)\right\} \mathrm{d}^{2} \mathbf{x}^{\prime},
$$

where the dummy integration variables are now denoted by $\mathbf{x}^{\prime}$, and the explicit dependence on $\mathbf{x}$ is introduced by the choice $\Omega:=\omega_{\Lambda}(\mathbf{x})$. We can then write $(\mathrm{C} .11)$ as

$$
\left(1-\mathrm{P}_{\Lambda}\right) \omega_{\varepsilon}=-\mathcal{E}\left[\omega_{\Lambda}, \omega_{\varepsilon}\right]
$$

If we now take $\left(1-\mathrm{P}_{\Lambda}\right)$ of (C.4) and use (C.13), we obtain [cf. (3.11)]

$$
\left(1-\mathrm{P}_{\Lambda}\right) \phi_{\varepsilon}=\left(1-\mathrm{P}_{\Lambda}\right) \psi_{\varepsilon}+G_{\Lambda}^{\prime} \mathcal{E}\left[\omega_{\Lambda}, \omega_{\varepsilon}\right]
$$

Using this, we can write (C.4) in the form [cf. (3.8)]

$$
\left(\Delta-F_{\Lambda}^{\prime} \mathrm{P}_{\Lambda}\right) \psi_{\varepsilon}=-F_{\Lambda}^{\prime} \mathrm{P}_{\Lambda} \phi_{\varepsilon}-\mathcal{E}\left[\omega_{\Lambda}, \omega_{\varepsilon}\right],
$$

which goes with the boundary conditions

$$
\psi_{\varepsilon}=\varepsilon b \quad \text { on } \partial D_{\Lambda} .
$$

Eqs. (C.7)-(C.15) form a system of nonlinear (partial pseudodifferential) equations which is to be solved for $\psi_{\varepsilon}$. Unlike the first-order equations in Section 3, their solution involves higher-order derivatives of $\Lambda$ since these are necessary to compute the time derivative on the right-hand side of (C.7). We could obtain an asymptotic solution of this system by iteration as follows.

Given $\psi_{\Lambda}$ and $\dot{\Lambda}$ along with all its derivatives, let $\psi^{[0]}=0$. Then for $n=0, \cdots$, we successively solve

$$
\begin{aligned}
& \left(\Delta-F_{\Lambda}^{\prime} \mathrm{P}_{\Lambda}\right) \varphi^{[n+1]}=\left(1-\Delta G_{\Lambda}^{\prime}\right)\left\{\varepsilon \partial_{\tau} \omega^{[n]}+\left[\psi^{[n]}, \omega^{[n]}\right]\right\} \quad \text { with } \varphi^{[n+1]}=\varepsilon\left[\psi_{\Lambda}, b\right] \quad \text { on } \partial D_{\Lambda}, \\
& {\left[\phi^{[n+1]}, \psi_{\Lambda}\right]=\mathrm{P}_{\Lambda} \varphi^{[n+1]}, \quad\left(\Delta-F_{\Lambda}^{\prime} \mathrm{P}_{\Lambda}\right) \psi^{[n+1]}=-F_{\Lambda}^{\prime} \mathrm{P}_{\Lambda} \phi^{[n+1]}-\mathcal{E}\left[\omega_{\Lambda}, \omega^{[n]}\right] \quad \text { with } \psi^{[n+1]}=\varepsilon b \quad \text { on } \partial D_{\Lambda} .}
\end{aligned}
$$

Due to the term $\partial_{t} \omega^{[n]}$ in $(\mathrm{C} .16)(\mathrm{a})$, which can be computed from $\dot{\Lambda}, \ldots, \mathrm{d}^{n} \Lambda / \mathrm{d} t^{n}$, this iteration is unlikely to converge as $\psi^{[n]}$ will lose differentiability as $n \rightarrow \infty$. However, given sufficiently small $\varepsilon$ and sufficiently smooth $\dot{\Lambda}$, arbitrarily high order can (formally) be achieved. It is clear from this construction that the streamfunction corrections $\psi^{[n]}$ are all "slaved" to the boundary motion, in the sense that if $\dot{\Lambda}=0$ in an open interval $I$ in time, $\psi^{[n]}=0$ for $t \in I$.

A couple of remarks are in order. First, $\psi^{[1]}=\varepsilon \psi^{(1)}$ found in Section 3, and similarly $\phi^{[1]}=\varepsilon \phi$; the relationships between higher-order terms $\psi^{(n)}$ and iterates $\psi^{[n]}$ are more complicated. Second, the foregoing development is formal; we leave the rigorous proof, which would involve bounding both the slaved streamfunctions $\psi^{[n]}$ and the remainder in appropriate function spaces, to a future work.

\section{Appendix D. Second-order terms in nearly axisymmetric flows}

At order $O\left(\delta^{2}\right), \rho_{2}$ is found from (5.9) to satisfy

$$
\frac{\psi_{0}^{\prime}}{r} \Delta \partial_{\sigma} \rho_{2}+2\left(\frac{\psi_{0}^{\prime}}{r}\right)^{\prime}\left(\partial_{r \sigma}^{2} \rho_{2}-\frac{1}{r} \partial_{\sigma} \rho_{2}\right)+\frac{2}{r}\left(r \chi_{2}^{\prime}\right)^{\prime}=2\left[\rho_{1}, \Delta\left[\rho_{1}, \psi_{0}\right]\right]-\left[\rho_{1},\left[\rho_{1}, \Delta \psi_{0}\right]\right]-\Delta\left[\rho_{1},\left[\rho_{1}, \psi_{0}\right]\right] .
$$


The boundary condition (5.12) can be written as

$$
\partial_{\sigma} \rho_{2}=\partial_{\sigma}\left(\partial_{r} \rho_{1} \partial_{\sigma} \rho_{1}\right)-\left(\partial_{\sigma} \rho_{1}\right)^{2}+\sum_{m}\left|\Lambda_{m}\right|^{2} \quad \text { at } r=1
$$

after some manipulations. The interest of this form is that, when (6.7) is taken into account, it is clearly consistent, with both sides having a vanishing $\sigma$-average. A solvability condition for (D.1) is obtained by averaging over $\sigma$, leading to

$$
\left(r \chi_{2}^{\prime}\right)^{\prime}=-\left(\frac{\psi_{0}^{\prime}}{2 \pi r} \int_{0}^{2 \pi}\left[\left(\partial_{r \sigma}^{2} \rho_{1}\right)^{2}+\frac{1}{r^{2}}\left(\partial_{\sigma \sigma}^{2} \rho_{1}\right)^{2}-\frac{2}{r} \partial_{\sigma} \rho_{1} \partial_{r \sigma}^{2} \rho_{1}\right] \mathrm{d} \sigma\right)^{\prime} .
$$

This equation determines $\chi_{2}$ uniquely up to an irrelevant arbitrary constant. When it is satisfied, (D.1) can be solved for $\rho_{2}$, yielding a solution in the form of a Fourier series

$$
\rho_{2}(r, \sigma)=\sum_{m} \hat{\rho}_{2, m}(r) \mathrm{e}^{\mathrm{i} m \sigma},
$$

with $\hat{\rho}_{2, m}^{*}=\hat{\rho}_{2,-m}$ and $\hat{\rho}_{2,0}=0$. The functions $\hat{\rho}_{2, m}$ satisfy an inhomogeneous version of (6.6) obtained from (D.2); clearly, they are quadratic in the $\Lambda_{m}$.

\section{Appendix E. Rotating ellipse}

Consider a fluid inside an ellipse with semi-axes $a$ and $b$ that is rotating around its centre with a (possibly time-dependent) angular velocity $\varepsilon \dot{\lambda}(\tau)$. The equation of the ellipse is given by

$$
B(\mathbf{x}, \tau)=\frac{\hat{x}_{1}^{2}}{a^{2}}+\frac{\hat{x}_{2}^{2}}{b^{2}}-1=0
$$

where

$$
\begin{aligned}
& \hat{x}_{1}=x \cos \lambda+y \sin \lambda, \\
& \hat{x}_{2}=-x \sin \lambda+y \cos \lambda
\end{aligned}
$$

are Cartesian coordinates in a frame rotating with angular velocity $\varepsilon \dot{\lambda}$. An exact solution for the fluid motion in such a rotating ellipse is provided by the uniform-vorticity flow with streamfunction

$$
\psi(\mathbf{x}, t)=K\left(\frac{\hat{x}_{1}^{2}}{a^{2}}+\frac{\hat{x}_{2}^{2}}{b^{2}}\right)+\frac{\varepsilon \dot{\lambda}\left(a^{2}-b^{2}\right)}{2\left(a^{2}+b^{2}\right)}\left(\hat{x}_{1}^{2}-\hat{x}_{2}^{2}\right)
$$

where $K$ is a constant. In this streamfunction, which can be verified directly to satisfy the boundary condition (2.5), the first term can be identified with $\psi^{(0)}$, the second with $\psi^{(1)}$, and there are no higher-order terms in $\varepsilon$ (see, e.g., [11, p. 421] for a derivation). Action-angle coordinates $(I, \theta)$ for this flow satisfy

$$
\begin{aligned}
& \hat{x}_{1}=\sqrt{2 I a / b} \cos \theta, \\
& \hat{x}_{2}=\sqrt{2 I b / a} \sin \theta .
\end{aligned}
$$

In terms of these variables, the streamfunction, or Hamiltonian, becomes

$$
\hat{H}(I, \theta)=\frac{2 K I}{a b}+\frac{\varepsilon \dot{\lambda} I}{a b}\left[\frac{a^{2}-b^{2}}{a^{2}+b^{2}}\left(a^{2} \cos ^{2} \theta-b^{2} \sin ^{2} \theta\right)-\left(a^{2} \cos ^{2} \theta+b^{2} \sin ^{2} \theta\right)\right] .
$$

In this expression, the last term between round brackets comes from the time dependence in the (canonical) transformation from $(x, y)$ to $(I, \theta)$; it simply corresponds to a rigid-body rotation with angular velocity $-\varepsilon \dot{\lambda}$. The geometric angle is derived by writing the evolution equation for $\theta$, averaging, then integrating in time. This gives

$$
\Delta \theta_{\text {geo }}=\frac{\Delta \lambda}{2 a b}\left[\frac{\left(a^{2}-b^{2}\right)^{2}}{a^{2}+b^{2}}-\left(a^{2}+b^{2}\right)\right],
$$

where $\Delta \lambda$ is the total angle rotated by the ellipse.

Note that the averaging is in fact unnecessary, since the Hamiltonian $\hat{H}$ does not depend on $\theta$ as a simplification of (E.1) indicates. We do not perform this simplification here, however, in order to retain the two terms separately, in (E.2). This facilitates the comparison with the general formalism of Section 4 . The first term in (E.2) stems from the correction in the streamfunction $\psi^{(1)}$ (which here corresponds to a potential flow), while the second stems from the slow time dependence of the leading-order flow; thus these two terms can be identified with the two contributions $\left\langle\mathbf{d} \hat{\Phi}^{\star}\right\rangle$ and $-\frac{1}{2}\left\langle\left[\hat{\Phi}^{\star} \wedge \hat{\Phi}^{\star}\right]\right\rangle$ in (4.25). 
We can use the exact formula (E.2) to verify the approximate results for slightly deformed axisymmetric flows obtained in Section 6. A rotating ellipse of small eccentricity is represented by the deformed disc $(6.1)$ with $\Lambda_{ \pm 2}(\tau)$ describing a unit circle in the complex plane and all the other $\Lambda_{m}$ equal to zero. The corresponding semi-axes are then

$$
a=1+2 \delta+O\left(\delta^{2}\right) \text { and } b=1-2 \delta+O\left(\delta^{2}\right),
$$

with the $O\left(\delta^{2}\right)$ corrections ensuring that $a b=1$. Introducing this into (E.2) and considering a full rotation $\Delta \lambda=2 \pi$ give the geometric angle

$$
\Delta \theta_{\mathrm{geo}}=\pi\left[32 \delta^{2}-\left(2+16 \delta^{2}\right)\right]+O\left(\delta^{3}\right)=-2 \pi+16 \pi \delta^{2}+O\left(\delta^{3}\right)
$$

An equivalent result is obtained from the developments in Section 6. Since the only non-zero parameter $\Lambda_{m}$ is $\Lambda_{ \pm 2},(6.14)$ reduces to

$$
\Delta \theta_{\text {geo }}=\delta^{2} f_{2}(r) \mathcal{A}_{2}+O\left(\delta^{3}\right) .
$$

The uniform vorticity corresponds to the limit $\alpha \rightarrow 2$ in (6.15), so that $\alpha_{2}=\beta_{2}=2, \gamma_{2}=1 / 2$, and $f_{2}(r)=8$, independent of $r$. Since a full rotation of the ellipse is obtained when $\Lambda_{2}(\tau)$ covers twice the unit circle, $\mathcal{A}_{2}=2 \pi$ and hence

$$
\Delta \theta_{\text {geo }}=16 \pi \delta^{2}+O\left(\delta^{3}\right)
$$

with contributions $32 \pi \delta^{2}$ and $-16 \pi \delta^{2}$ from $\left\langle\mathbf{d} \hat{\Phi}^{*}\right\rangle$ and $-\frac{1}{2}\left\langle\left[\hat{\Phi}^{*}, \hat{\Phi}^{*}\right]\right\rangle$, respectively. The discrepancy of $-2 \pi$ when compared with (E.3) results from a different definition of the angle $\theta$, which is measured from an axis rotating with the ellipse in the calculation leading to (E.3) while it is measured from a fixed axis in Section 6.

\section{References}

[1] V.I. Arnold, Conditions for nonlinear stability of stationary plane curvilinear flows of an ideal fluid, Dokl. Akad. Nauk SSSR 162 (1965) 975-978 (in Russian); English translation in: Soviet Math. 6, 773-777.

[2] V.I. Arnold, On an a priori estimate in the theory of hydrodynamical stability, Izv. Vyssh. Uchebn. Zaved. Matematika 53 (1966) 3-5 (in Russian); English translation in: Amer. Math. Soc. Transl. Ser. 2, 79, 267-269.

[3] V.I. Arnold, Mathematical Methods of Classical Mechanics, 2nd ed., Springer-Verlag, 1989.

[4] V.I. Arnol'd, B.A. Khesin, Topological Methods in Hydrodynamics, Springer-Verlag, 1998.

[5] M.V. Berry, Classical adiabatic angles and quantal adiabatic phase, J. Phys. A: Math. Gen. 18 (1) (1985) $15-27$.

[6] P.G. Drazin, W.H. Reid, Hydrodynamic Stability, Cambridge Univ. Press, 1981.

[7] T. Frankel, The Geometry of Physics, 2nd ed., Springer-Verlag, 2004.

[8] D. Gilbarg, N.S. Trudinger, Elliptic Partial Differential Equations of Second Order, Springer-Verlag, 1977.

[9] J.H. Hannay, Angle variable holonomy in adiabatic excursion of an integrable Hamiltonian, J. Phys. A: Math. Gen. 18 (2) (1985) $221-230$.

[10] D.D. Holm, J.E. Marsden, T.S. Ratiu, A. Weinstein, Nonlinear stability of fluid and plasma equilibria, Phys. Rep. 123 (1-2) (1985) 1-116.

[11] H. Jeffreys, B. Jeffreys, Methods of Mathematical Physics, 3rd ed., Cambridge Univ. Press, 1974.

[12] L.D. Landau, E.M. Lifshitz, Mechanics, Pergamon, 1960.

[13] A.J. Lichtenberg, M.A. Lieberman, Regular and Chaotic Dynamics, 2nd ed., Springer-Verlag, 1992.

[14] J.E. Marsden, R. Montgomery, T.S. Ratiu, Reduction, symmetry and phases in mechanics, Mem. AMS 436 (1990) 110.

[15] S.A. Maslowe, Critical layers in shear flows, Ann. Rev. Fluid Mech. 18 (1986) 405-432.

[16] R. Montgomery, The connection whose holonomy is the classical adiabatic angles of Hannay and Berry and its generalization to the non-integrable case, Commun. Math. Phys. 120 (1988) 269-294.

[17] P.J. Morrison, Hamiltonian description of the ideal fluid, Rev. Modern Phys. 70 (2) (1998) 467-521.

[18] P.G. Saffman, Vortex Dynamics, Cambridge Univ. Press, 1992.

[19] R. Salmon, Hamiltonian fluid mechanics, Ann. Rev. Fluid Mech. 20 (1988) 225-256.

[20] A. Shapere, F. Wilczek, Gauge kinematics of deformable bodies, in: A. Shapere, F. Wilczek (Eds.), Geometric Phases in Physics, World Scientific, 1989, pp. 449-459.

[21] A. Shapere, F. Wilczek, Geometry of self-propulsion at low Reynolds number, J. Fluid Mech. 198 (1989) $557-585$.

[22] B.N. Shashikanth, P.K. Newton, Vortex motion and the geometric phase. Part I. Basic configurations and asymptotics, J. Nonlinear Sci. 8 (1997) 183-214.

[23] B.N. Shashikanth, P.K. Newton, Vortex motion and the geometric phase. Part II. Slowly varying spiral structures, J. Nonlinear Sci. 9 (1999) $233-254$.

[24] K. Stewartson, The evolution of the critical layer of a Rossby wave, Geophys. Astrophys. Fluid Dynamics 9 (1978) 185-200.

[25] D. Wirosoetisno, J. Vanneste, Persistence of steady flows of a two-dimensional perfect fluid in deformed domains, Nonlinearity 18 (2005) $2657-2680$. 